\title{
Associated Hermite Polynomials Related to Parabolic Cylinder Functions
}

\author{
Alfred Wünsche \\ Max-Planck-Group “Nonclassical Radiation” at Institut für Physik, Humboldt-Universität Berlin, \\ Berlin, Germany \\ Email: alfred.wuensche@physikhu-berlin.de
}

How to cite this paper: Wünsche, $A$ (2019) Associated Hermite Polynomials Related to Parabolic Cylinder Functions. Advances in Pure Mathematics, 9, 15-42. https://doi.org/10.4236/apm.2019.91002

Received: November 26, 2018

Accepted: January 20, 2019

Published: January 23, 2019

Copyright (c) 2019 by author(s) and Scientific Research Publishing Inc. This work is licensed under the Creative Commons Attribution International License (CC BY 4.0).

http://creativecommons.org/licenses/by/4.0/

\section{cc) (i) Open Access}

\begin{abstract}
In analogy to the role of Lommel polynomials $\mathrm{R}_{n}^{v}(z)$ in relation to Bessel functions $\mathrm{J}_{v}(z)$ the theory of Associated Hermite polynomials in the scaled form $\mathrm{He}_{n}^{v}(z)$ with parmeter $v$ to Parabolic Cylinder functions $\mathrm{D}_{v}(z)$ is developed. The group-theoretical background with the 3-parameter group of motions $M(2)$ in the plane for Bessel functions and of the Heisenberg-Weyl group $W(2)$ for Parabolic Cylinder functions is discussed and compared with formulae, in particular, for the lowering and raising operators and the eigenvalue equations. Recurrence relations for the Associated Hermite polynomials and for their derivative and the differential equation for them are derived in detail. Explicit expressions for the Associated Hermite polynomials with involved Jacobi polynomials at argument zero are given and by means of them the Parabolic Cylinder functions are represented by two such basic functions.
\end{abstract}

\section{Keywords}

Bessel Functions, Lommel Polynomials, Parabolic Cylinder Functions,

Associated Hermite Polynomials, Jacobi polynomials, Recurrence Relations,

Lowering and Raising Operators, Heisenberg-Weyl Group, Motion Group of

Plane, Irreducible Representations

\section{Introduction}

Let us motivate our intentions by an analogy of Bessel functions $\mathrm{J}_{v}(z)$ to Parabolic Cylinder functions $\mathrm{D}_{v}(z)$. Both sets of functions satisfy a certain second-order differential equation and a certain 3-term recurrence relation. The 3-term recurrence relation for the Bessel functions in the form $\mathrm{J}_{v+n}(z)$ pro- 
vides the possibility to express it successively by the sum of two Bessel functions with lower indices $\mathrm{J}_{v+m}(z)$ and $\mathrm{J}_{v+m-1}(z),(m<n)$, and we may continue this up to the case where we have presented $\mathrm{J}_{v+n}(z)$ by a superposition of two Bessel functions $\mathrm{J}_{v}(z)$ and $\mathrm{J}_{v-1}(z)$ considered as our "basic" functions of the form

$$
\mathrm{J}_{v+n}(z)=\mathrm{R}_{n}^{v}(z) \mathrm{J}_{v}(z)-\mathrm{R}_{n-1}^{v+1}(z) \mathrm{J}_{v-1}(z), \quad(n=0,1,2, \cdots, v \in \mathbb{R}),
$$

with coefficients $\mathrm{R}_{n}^{v}(z)$ which are polynomials of $n$-th degree of variable $z^{-1}$ called Lommel polynomials. Their explicit form is known [1] (cited according to Watson [2]). We find this in Watson [2] (from p. 294 on in very detailed form) and in Bateman and Erdélyi [3] (chap. 7.5.2, p. 43) with the explicit formula for the Lommel polynomials ${ }^{1}([\mu]$ means integer part of $\mu$ )

$$
\mathrm{R}_{n}^{v}(z) \equiv \sum_{k=0}^{\left[\frac{n}{2}\right]} \frac{(-1)^{k}(n-k) !(n+v-k-1) !}{k !(n-2 k) !(v+k-1) !}\left(\frac{2}{z}\right)^{n-2 k} .
$$

Whereas Lommel derives his polynomials by a somewhat cumbersome induction ([2], chap. 9.61.) Watson derives the Lommel polynomials from some (Laurent) series of products of Bessel functions that according to his statement is simpler. Furthermore, he discusses in chap. 9.7 (from p. 303 on) a related function $g_{n, v}\left(\frac{z^{2}}{4}\right) \equiv\left(\frac{z}{2}\right)^{n} \mathrm{R}_{n, v}(z)$ which in similar form was introduced by Hurwitz [4] (cited on page 302 in [2]).

By an analogous process one may relate the Parabolic Cylinder functions $\mathrm{D}_{v+n}(z)$ to the sum of two Parabolic Cylinder functions $\mathrm{D}_{v}(z)$ and $\mathrm{D}_{v-1}(z)$ considered as our "basic" functions of the form

$$
\mathrm{D}_{v+n}(z)=\mathrm{He}_{n}^{v}(z) \mathrm{D}_{v}(z)-v \mathrm{He}_{n-1}^{v+1}(z) \mathrm{D}_{v-1}(z),
$$

with polynomials $\mathrm{He}_{n}^{v}(z)$ of $n$-th degree in variable $z$ which possess the explicit form

$$
\begin{aligned}
\mathrm{He}_{n}^{v}(z) & =\sum_{k=0}^{\left[\frac{n}{2}\right]} \frac{(n-k) !}{(n-2 k) !}\left(\frac{(-1)^{k}}{(v-1) !} \sum_{j=0}^{k} \frac{(v-1+j) !}{j !(k-j) ! 2^{k-j}}\right) z^{n-2 k} \\
& =\sum_{k=0}^{\left[\frac{n}{2}\right]} \frac{(n-k) !}{(n-2 k) !} \mathrm{P}_{k}^{(-v-k, n+v-k)}(0) z^{n-2 k},
\end{aligned}
$$

where $\mathrm{P}_{k}^{(\alpha, \beta)}(u)$ denotes the Jacobi polynomials introduced in this form by Szegö [5] and here taken for argument $u=0$. The polynomials (1.4) are associated to the Hermite polynomials in a scaled form and are for $v=0$ identical with the scaled Hermite polynomials usually denoted by $\operatorname{He}_{n}(z)$ that means $\mathrm{He}_{n}(z) \equiv \operatorname{He}_{n}^{0}(z)$. We call them Associated Hermite polynomials (scaled for applications in connection with the Parabolic Cylinder functions $\left.\mathrm{D}_{v}(z)\right)$. They ${ }^{1}$ We change slightly the standard notation of the Lommel polynomials [1] [2] [3] according to $\mathrm{R}_{n, v}(z) \rightarrow \mathrm{R}_{n}^{v}(z)$ for reason which is better to feel after the development of the analogous formalism for Parabolic Cylinder functions. 
were introduced together with some of their basic relations in [6].

One of the possible applications of Parabolic Cylinder functions is in quantum mechanics for the calculation of eigenfunctions of squeezing and rotation operators in the two-dimensional phase plane that means for the general quadratic combinations of a pair of boson annihilation and creation operators and, more generally, for several pairs of boson operators. This is also connected with the reduction to two possible normal forms and one degenerate form of quadratic combinations of such operators. For squeezing operators the eigenfunctions are not normalizable and up to now are little known.

The notions "Associated Hermite polynomials" and "Generalized Hermite polynomials" are not used in fully unique sense in literature, e.g., [7] [8]. However, our notation $\operatorname{He}_{n}^{v}(z)$ for them seems to be unique and specifies them. Our "Associated Hermite polynomials" are related to the Parabolic Cylinder functions in the analogous way as Lommel polynomials are related to Bessel functions. They are, in general, not orthogonal polynomials and satisfy a 4-th order differential equation.

In the following Sections we develop more systematically some formalism for the Parabolic Cylinder functions in connection with their Associated Hermite polynomials. Later, after this we will come back again to the analogies between Bessel functions and Parabolic Cylinder functions with discussion of the group-theoretical background.

\section{The Parabolic Cylinder Functions in the Form $\mathbf{D}_{v}(z)$}

The Weber equation with parameter $v \in \mathbb{R}$

$$
\left\{\frac{\partial^{2}}{\partial z^{2}}-\frac{z^{2}}{4}+v+\frac{1}{2}\right\} y(v ; z)=0
$$

with important application in physics (e.g., quantum mechanics) is satisfied, for example, by the following two independent solutions

$$
\begin{aligned}
& y_{1}(v ; z)=\exp \left(-\frac{z^{2}}{4}\right){ }_{1} \mathrm{~F}_{1}\left(-\frac{v}{2} ; \frac{1}{2} ; \frac{z^{2}}{2}\right), \\
& y_{2}(v ; z)=\exp \left(-\frac{z^{2}}{4}\right) z_{1} \mathrm{~F}_{1}\left(\frac{1-v}{2} ; \frac{3}{2} ; \frac{z^{2}}{2}\right),
\end{aligned}
$$

with the Wronski determinant

$$
W\left(y_{1}, y_{2}\right)(v ; z) \equiv y_{1}(v ; z) \frac{\partial}{\partial z} y_{2}(v ; z)-y_{2}(v ; z) \frac{\partial}{\partial z} y_{1}(v ; z)=1 .
$$

It is independent of variable $z$ due to differential Equation (2.1) with no first-order derivative and with a second-order derivative without a coefficient in front depending on $z$ as it is easily to derive. In addition, it is independent on parameter $v$ that is a special property of the two solutions (2.2). Due to independence of $z$ we obtain the Wronski determinant (2.3) setting, for example, $z=0$. 
From the two linearly independent solutions $y_{1}(v ; z)$ and $y_{2}(v ; z)$ one constructs by superposition as definition $y(v ; z) \equiv \mathrm{D}_{v}(z)$ the following solution of the Weber Equation (2.1)

$$
\begin{aligned}
\mathrm{D}_{v}(z) \equiv & 2^{\frac{v}{2}} \exp \left(-\frac{z^{2}}{4}\right)\left\{\frac{\left(-\frac{1}{2}\right) !}{\left(-\frac{1+v}{2}\right) !}, \mathrm{F}_{1}\left(-\frac{v}{2} ; \frac{1}{2} ; \frac{z^{2}}{2}\right)\right. \\
& \left.+\frac{z}{\sqrt{2}} \frac{\left(-\frac{3}{2}\right) !}{\left(-1-\frac{v}{2}\right) !}{ }_{1} \mathrm{~F}_{1}\left(\frac{1-v}{2} ; \frac{3}{2} ; \frac{z^{2}}{2}\right)\right\} \\
= & 2^{\frac{v}{2}} \exp \left(\frac{z^{2}}{4}\right)\left\{\frac{\left(-\frac{1}{2}\right) !}{\left(-\frac{1+v}{2}\right) !}, \mathrm{F}_{1}\left(\frac{1+v}{2} ; \frac{1}{2} ;-\frac{z^{2}}{2}\right)\right. \\
& \left.+\frac{z}{\sqrt{2}} \frac{\left(-\frac{3}{2}\right) !}{\left(-1-\frac{v}{2}\right) !}{ }_{1} \mathrm{~F}_{1}\left(1+\frac{v}{2} ; \frac{3}{2} ;-\frac{z^{2}}{2}\right)\right\},
\end{aligned}
$$

where we used in the second representation the Kummer transformation of the Confluent Hypergeometric function (e.g., [9], (chap. (6.3, Equation (7)))

$$
{ }_{1} \mathrm{~F}_{1}(a ; c ; z)=\mathrm{e}^{z}{ }_{1} \mathrm{~F}_{1}(c-a ; c ;-z),
$$

and with $\left(-\frac{1}{2}\right) !=\sqrt{\pi},\left(-\frac{3}{2}\right) !=-2 \sqrt{\pi}$. A second independent solution of the Weber equation can be constructed in various ways from superpositions of the kind

$$
f(v ; z)=\alpha \mathrm{D}_{v}(z)+\beta \mathrm{D}_{v}(-z)+\gamma \mathrm{D}_{-v-1}(\mathrm{i} z)+\delta \mathrm{D}_{-v-1}(-\mathrm{i} z),
$$

with coefficients $(\alpha, \beta, \gamma, \delta)$ but maximally only two of the four basic functions $\left(\mathrm{D}_{v}( \pm z), \mathrm{D}_{-v-1}( \pm \mathrm{i} z)\right)$ can be linearly independent solutions of the Weber equation. For example

$$
\mathrm{D}_{v}(z)=\frac{v !}{\sqrt{2 \pi}}\left\{\exp \left(\mathrm{i} v \frac{\pi}{2}\right) \mathrm{D}_{-v-1}(\mathrm{i} z)+\exp \left(-\mathrm{i} v \frac{\pi}{2}\right) \mathrm{D}_{-v-1}(-\mathrm{i} z)\right\},
$$

is such a relation between three of these functions [3] (chap. 8.2. (6)).

Instead of $\mathrm{D}_{v}(z)$ other authors use a fully equivalent form of basic Parabolic Cylinder function with notation $\mathrm{U}(\mu, z)$ related to $\mathrm{D}_{v}(z)$ as follows (e.g., Temme [10] in chap. 12 of NIST Handbook [11] and also J. Miller [12] in the older Handbook by the editors Abramowitz and Stegun [13])

$$
\mathrm{D}_{v}(z) \equiv \mathrm{U}\left(-v-\frac{1}{2}, z\right), \Leftrightarrow \mathrm{U}(\mu, z) \equiv \mathrm{D}_{-\mu-\frac{1}{2}}(z) .
$$

A few but very important special cases of these definitions which can be also 
expressed by other Special functions are [10] (p. 309)

$$
\begin{gathered}
\mathrm{D}_{\frac{1}{2}}(z)=\mathrm{U}(-1, z)=\frac{z^{\frac{3}{2}}}{2 \sqrt{2 \pi}}\left(\mathrm{K}_{\frac{3}{4}}\left(\frac{z^{2}}{4}\right)+\mathrm{K}_{\frac{1}{4}}\left(\frac{z^{2}}{4}\right)\right), \\
\mathrm{D}_{-\frac{1}{2}}(z)=\mathrm{U}(0, z)=\sqrt{\frac{z}{2 \pi}} \mathrm{K}_{\frac{1}{4}}\left(\frac{z^{2}}{4}\right), \\
\mathrm{D}_{-\frac{3}{2}}(z)=\mathrm{U}(1, z)=\frac{z^{\frac{3}{2}}}{\sqrt{2 \pi}}\left(\mathrm{K}_{\frac{3}{4}}\left(\frac{z^{2}}{4}\right)-\mathrm{K}_{\frac{1}{4}}\left(\frac{z^{2}}{4}\right)\right),
\end{gathered}
$$

and (e.g., [10], p. 309, 12.7 (iii))

$$
\begin{gathered}
\mathrm{D}_{0}(z)=\mathrm{U}\left(-\frac{1}{2}, z\right)=\exp \left(-\frac{z^{2}}{4}\right), \\
\mathrm{D}_{-1}(z)=\mathrm{U}\left(\frac{1}{2}, z\right)=\exp \left(\frac{z^{2}}{4}\right) \sqrt{\frac{\pi}{2}}\left(1-\operatorname{Erf}\left(\frac{z}{\sqrt{2}}\right)\right) \\
\equiv \exp \left(\frac{z^{2}}{4}\right) \sqrt{\frac{\pi}{2}} \operatorname{Erfc}\left(\frac{z}{\sqrt{2}}\right) .
\end{gathered}
$$

Herein, $\mathrm{K}_{v}(u)$ is a standard notation for a category of Bessel functions (e.g., [3], chap. 7.2.2. Equation (13), [11], p. 251)

$$
\mathrm{K}_{v}(u) \equiv \frac{\pi}{2} \frac{\mathrm{I}_{-v}(u)-\mathrm{I}_{v}(u)}{\sin (v \pi)},
$$

and $\operatorname{Erf}(u)$ denotes the Error function defined by ${ }^{2}$

$$
\operatorname{Erf}\left(\frac{z}{a}\right) \equiv \frac{2}{\sqrt{\pi a^{2}}} \int_{0}^{z} \mathrm{~d} t \exp \left(-\frac{t^{2}}{a^{2}}\right), \quad(a>0) .
$$

The function $\operatorname{Erfc}(u) \equiv 1-\operatorname{Erf}(u)$ is the Complementary Error function.

\section{Series Representations of the Parabolic Cylinder Functions}

If we insert in (2.4) the well-known Taylor series of the Confluent Hypergeometric function

$$
{ }_{1} \mathrm{~F}_{1}(a ; c ; z) \equiv \frac{(c-1) !}{(a-1) !} \sum_{k=0}^{\infty} \frac{(k+a-1) !}{(k+c-1) !} \frac{z^{k}}{k !}
$$

then we may derive two different series representations of the Parabolic Cylinder functions $\mathrm{D}_{v}(z)$.

${ }^{2}$ Using Erdélyi, Vol. 2 [3] (chap. 8 together with chap. 9.9.) one has to pay attention that in relations to the Error function $\operatorname{Erf}(x)$ the last is defined there under the same notation without the factor $\frac{2}{\sqrt{\pi}}$ in comparison to the modern definition (2.12) which is here given and which is used in Program "Mathematica". 
If we use the first representation in (2.4) we obtain a series representation which can be written in the following compact form

$$
\mathrm{D}_{v}(z)=\exp \left(-\frac{z^{2}}{4}\right) 2^{\frac{v}{2}} \sqrt{\pi} \sum_{k=0}^{\infty} \frac{(-1)^{k}}{k !\left(\frac{k-v-1}{2}\right) !=\text { Poch. }}\left(\frac{(-v)_{k}}{\sqrt{2}}\right)^{k},
$$

where $(-v)_{k}$ denotes the Pochhammer symbol according to the definition

$$
(a)_{k} \equiv \frac{(k+a-1) !}{(a-1) !}, \Rightarrow(-v)_{k} \equiv \frac{(k-v-1) !}{(-v-1) !}=\frac{(-1)^{k} v !}{(v-k) !} .
$$

Using the first of the given representations of the Pochhammer symbol $(-v)_{k}$ one finds from (3.2)

$$
\begin{aligned}
\mathrm{D}_{v}(z) & =\exp \left(-\frac{z^{2}}{4}\right) \frac{2^{\frac{v}{2}} \sqrt{\pi}}{(-v-1) !} \sum_{k=0}^{\infty} \frac{(-1)^{k}(k-v-1) !}{k !\left(\frac{k-v-1}{2}\right) !}\left(\frac{z}{\sqrt{2}}\right)^{k} \\
& =\exp \left(-\frac{z^{2}}{4}\right) \frac{1}{2^{\frac{v}{2}+1}(-v-1) !} \sum_{k=0}^{\infty} \frac{(-1)^{k}\left(\frac{k-v}{2}-1\right) !}{k !}(\sqrt{2} z)^{k} .
\end{aligned}
$$

These representations fail to act in the important special cases of non-negative integers $v=n=0,1,2, \cdots$ due to infinities in numerators and denominator. Using the second of the given representations of the Pochhammer symbol $(-v)_{k}$ one finds from (3.2) alternatively

$$
\mathrm{D}_{v}(z)=\exp \left(-\frac{z^{2}}{4}\right) 2^{\frac{v}{2}} \sqrt{\pi} \sum_{k=0}^{\infty} \frac{v !}{k !\left(\frac{k-v-1}{2}\right) !(v-k) !}\left(\frac{z}{\sqrt{2}}\right)^{k} .
$$

This representation fails to provide the result without limiting transitions for negative integers $v=-n=-1,-2,-3, \cdots$ due to infinities in numerator and denominator. This is the main reason why we gave in (3.2) the representation by the Pochhammer symbol ${ }^{3}$.

If we use the second representation in (2.4) we obtain from the series representation of the Confluent Hypergeometric function (3.1) a series representation of $\mathrm{D}_{v}(z)$ which can be written in the following form $\left(\left(-\frac{1}{2}\right)=\sqrt{\pi}\right)$

$$
\mathrm{D}_{v}(z)=\exp \left(\frac{z^{2}}{4}\right) \frac{2^{\frac{v}{2}}}{\left(-\frac{1}{2}\right) !} \sum_{k=0}^{\infty} \cos \left(\frac{k-v}{2} \pi\right)\left(\frac{k+v-1}{2}\right) ! \frac{(\sqrt{2} z)^{k}}{k !}
$$

or separated in the even and odd part

${ }^{3}$ The Pochhammer symbol $(a)_{k}$ is programmed in "Mathematica" as "Pochhammer [a,k]" in a way that it does not fail also in the mentioned special cases of failure of formulae (3.4) and (3.5). 


$$
\begin{aligned}
\mathrm{D}_{v}(z)= & \exp \left(\frac{z^{2}}{4}\right) \frac{2^{\frac{v}{2}}}{\left(-\frac{1}{2}\right) !}\left\{\cos \left(v \frac{\pi}{2}\right) \sum_{l=0}^{\infty}(-1)^{l}\left(l+\frac{v-1}{2}\right) ! \frac{(\sqrt{2} z)^{2 l}}{(2 l) !}\right. \\
& \left.+\sin \left(v \frac{\pi}{2}\right) \sum_{l=0}^{\infty}(-1)^{l}\left(l+\frac{v}{2}\right) ! \frac{(\sqrt{2} z)^{2 l+1}}{(2 l+1) !}\right\}
\end{aligned}
$$

We gave here only Taylor series for $\exp \left(\mp \frac{z^{2}}{4}\right) \mathrm{D}_{v}(z)$ since pure Taylor series of $\mathrm{D}_{v}(z)$ are complicated and not favorable for applications.

The basic Parabolic Cylinder functions $\mathrm{D}_{v}(z)$ were introduced in a way that they are vanishing for real $z=x$ in the limit $x \rightarrow+\infty$ for arbitrary real indices $v \in \mathbb{R}$.

\section{Lowering and Raising Operators for the Indices of the Parabolic Cylinder Functions and Derivatives and Recurrence Relations}

Practically, from every of the given explicit representations of $\mathrm{D}_{v}(z)$ in last Sections 2 and 3 one can derive formulae for the differentiation of this function. The simplest form they take on if we differentiate $\exp \left( \pm \frac{z^{2}}{4}\right) \mathrm{D}_{v}(z)$ and find or may check the known formulae (e.g., [3] [10])

$$
\begin{gathered}
\frac{\partial}{\partial z} \exp \left(\frac{z^{2}}{4}\right) \mathrm{D}_{v}(z)=v \exp \left(\frac{z^{2}}{4}\right) \mathrm{D}_{v-1}(z), \\
\frac{\partial}{\partial z} \exp \left(-\frac{z^{2}}{4}\right) \mathrm{D}_{v}(z)=-\exp \left(-\frac{z^{2}}{4}\right) \mathrm{D}_{v+1}(z) .
\end{gathered}
$$

These relations can be written

$$
\begin{aligned}
& \left(\frac{z}{2}+\frac{\partial}{\partial z}\right) \mathrm{D}_{v}(z) \equiv A \mathrm{D}_{v}(z)=v \mathrm{D}_{v-1}(z), \\
& \left(\frac{z}{2}-\frac{\partial}{\partial z}\right) \mathrm{D}_{v}(z) \equiv A^{\dagger} \mathrm{D}_{v}(z)=\mathrm{D}_{v+1}(z) .
\end{aligned}
$$

We call $\frac{z}{2}+\frac{\partial}{\partial z}$ the lowering and $\frac{z}{2}-\frac{\partial}{\partial z}$ the raising operator for the Parabolic Cylinder functions $\mathrm{D}_{v}(z)$ and denote them by $A$ and $A^{\dagger}$, respectively

$$
A \equiv \frac{z}{2}+\frac{\partial}{\partial z}=\frac{z}{2}+\mathrm{i}\left(-\mathrm{i} \frac{\partial}{\partial z}\right), \quad A^{\dagger} \equiv \frac{z}{2}-\frac{\partial}{\partial z}=\frac{z}{2}-\mathrm{i}\left(-\mathrm{i} \frac{\partial}{\partial z}\right),
$$

The operators $A$ and $A^{\dagger}$ are Hermitean adjoint ones to each other in spaces of functions of real variables $z$ and they satisfy the following commutation relations

$$
\left[A, A^{\dagger}\right] \equiv\left[\frac{z}{2}+\frac{\partial}{\partial z}, \frac{z}{2}-\frac{\partial}{\partial z}\right]=1, \quad[A, 1]=\left[A^{\dagger}, 1\right]=0
$$


These are the commutation relations for a Heisenberg-Weyl Lie algebra $w(2)$ (corresponding to Lie group $W(2))$ in representation by a pair of boson annihilation and creation operator in a space of functions $f(z)$ and each set of the Parabolic Cylinder functions $\mathrm{D}_{v \pm n}(z),(n=0,1,2, \cdots)$ forms a basis of a countably infinite irreducible unitary representation of this Lie algebra characterized by the index $v$ within the interval $0 \leq v<1$. The simplest eigenvalue equations for arbitrary $v$ are

$$
A^{\dagger} A \mathrm{D}_{v}(z)=v \mathrm{D}_{v}(z) .
$$

However, only the functions $\mathrm{D}_{n}(z)$ with $n=0,1,2, \cdots$ from these eigenfunctions prove themselves to be normalizable. Only for the irreducible representation with index $v=0$ with the basis functions

$\mathrm{D}_{ \pm n}(z),(n=0,1,2, \cdots)$ onto functions $\mathrm{D}_{n}(z)$ with $n \geq 0$ acting onto them with the lowering operator $A$ it arises the impression that this representation breaks down with $n=0$ for $A \mathrm{D}_{0}(z)=0$ but coming from below and acting with the raising operator $A^{\dagger}$ onto functions $\mathrm{D}_{n}(z)$ with $n<0$ we do not have a breakdown at $n=0$ and all basis functions $\mathrm{D}_{ \pm n}(z)$ are related also in this representation.

From (4.2) we find first the derivative of $\mathrm{D}_{v}(z)$

$$
\frac{\partial}{\partial z} \mathrm{D}_{v}(z)=\frac{1}{2}\left(v \mathrm{D}_{v-1}(z)-\mathrm{D}_{v+1}(z)\right),
$$

and second, the 3-term recurrence relation

$$
\mathrm{D}_{v+1}(z)-z \mathrm{D}_{v}(z)+v \mathrm{D}_{v-1}(z)=0 .
$$

Using the following general disentanglement of linear combinations of operators $z$ and $\frac{\partial}{\partial z}[14]$

$$
\left(\alpha \frac{z}{2}-\beta \frac{\partial}{\partial z}\right)^{n}=\sum_{k=0}^{n} \frac{(-1)^{k} n !}{k !(n-k) !}\left(\frac{\sqrt{\alpha \beta}}{2}\right)^{n-k} \mathrm{H}_{n-k}\left(\frac{\alpha z}{2 \sqrt{\alpha \beta}}\right)\left(\beta \frac{\partial}{\partial z}\right)^{k},
$$

which can be proved by complete induction ${ }^{4}$ one finds from (4.2) (see also (3.3) for representation with the Pochhammer symbol)

$$
\begin{aligned}
\mathrm{D}_{v+n}(z) & =\left(\frac{z}{2}-\frac{\partial}{\partial z}\right)^{n} \mathrm{D}_{v}(z) \\
& =\sum_{k=0}^{n} \frac{(-1)^{k} n !}{k !(n-k) !}\left(\frac{1}{2}\right)^{n-k} \mathrm{H}_{n-k}\left(\frac{z}{2}\right) \frac{\partial^{k}}{\partial z^{k}} \mathrm{D}_{v}(z), \\
\mathrm{D}_{v-n}(z) & =\frac{(v-n) !}{v !}\left(\frac{z}{2}+\frac{\partial}{\partial z}\right)^{n} \mathrm{D}_{v}(z) \\
= & \underbrace{\frac{(v-n) !}{v !}(-1)^{n}}_{\equiv \frac{1}{(-v)_{n}}} \sum_{k=0}^{n} \frac{n !}{k !(n-k) !}\left(\frac{\mathrm{i}}{2}\right)^{n-k} \mathrm{H}_{n-k}\left(\mathrm{i} \frac{z}{2}\right) \frac{\partial^{k}}{\partial z^{k}} \mathrm{D}_{v}(z) .
\end{aligned}
$$

${ }^{4}$ For $\sqrt{\alpha \beta}$ in this formula can be chosen an arbitrary sign but it has to be the same in all parts of the right-hand side. 
The second formula fails to act for nonnegative integers $v=0,1, \cdots$ in cases of $v-n<0$.

Alternatively we find from (4.1)

$$
\begin{gathered}
\mathrm{D}_{v+n}(z)=(-1)^{n} \exp \left(\frac{z^{2}}{4}\right) \frac{\partial^{n}}{\partial z^{n}} \exp \left(-\frac{z^{2}}{4}\right) \mathrm{D}_{v}(z), \\
\mathrm{D}_{v-n}(z)=\frac{(v-n) !}{v !} \exp \left(-\frac{z^{2}}{4}\right) \frac{\partial^{n}}{\partial z^{n}} \exp \left(\frac{z^{2}}{4}\right) \mathrm{D}_{v}(z) .
\end{gathered}
$$

Inserting in the first of these formulae $v=0$ and in the second $v=-1$ and using (2.10) we find known formulae for the Parabolic Cylinder functions $\mathrm{D}_{v}(z)$ with positive and negative integer $v$.

The formulae up to this point are more or less known but are necessary to make the paper widely self-contained. In next Section we show that we can generate the Parabolic Cylinder functions from two basic such functions by multiplication with a certain kind of polynomials which generalize the Hermite polynomials $\mathrm{H}_{n}(z)$ in the scaled form usually denoted by $\mathrm{He}_{n}(z)$.

\section{Representation of Parabolic Cylinder Functions by Two Neighbored Basic Ones with the Recurrence Relation}

Applying the recurrence relations $(4.7)$ to $\mathrm{D}_{v+n}(z)$ one may successively reduce it to a superposition of two basic functions $\mathrm{D}_{v}(z)$ and $\mathrm{D}_{v-1}(z)$ with coefficients depending on variable $z$ of the form

$$
\mathrm{D}_{v+n}(z)=\mathrm{He}_{n}^{v}(z) \mathrm{D}_{v}(z)-v \mathrm{He}_{n-1}^{v+1}(z) \mathrm{D}_{v-1}(z), \quad(n=0,1,2, \cdots ; v \in \mathbb{R}),
$$

where $\operatorname{He}_{n}^{v}(z)$ are sequences of polynomials of degree $n$ depending on $v$ as a parameter. For reason which we see below we call them associated Hermite polynomials. In the same way by the same recurrence relations (4.7) one may successively reduce $\mathrm{D}_{v-n}(z)$ to a superposition of the two basic functions $\mathrm{D}_{v}(z)$ and $\mathrm{D}_{v+1}(z)$ with coefficients depending on variable $z$ of the form (see also (3.3) for Pochhammer symbol $\left.(-v)_{n}\right)$

$$
\mathrm{D}_{v-n}(z)=\underbrace{\frac{(v-n) !}{v !}}_{=\frac{(-1)^{n}}{(-v)_{n}}}\left\{(-\mathrm{i})^{n} \mathrm{He}_{n}^{-v-1}(\mathrm{i} z) \mathrm{D}_{v}(z)-(-\mathrm{i})^{n-1} \mathrm{He}_{n-1}^{-v}(\mathrm{i} z) \mathrm{D}_{v+1}(z)\right\} \text {. }
$$

The general form of the polynomials $\operatorname{He}_{n}^{v}(z)$ of degree $n$ is the following

$$
\begin{aligned}
\mathrm{He}_{n}^{v}(z) & =\sum_{k=0}^{\left[\frac{n}{2}\right]} \frac{(-1)^{k}}{(n-2 k) !}\left(\sum_{j=0}^{k} \frac{(n-j) !(v-1+j) !}{j !(k-j) !(v-1) ! 2^{k-j}}\right) z^{n-2 k} \\
& =\sum_{k=0}^{\left[\frac{n}{2}\right]} \frac{(-1)^{k}}{(n-2 k) !}\left(\sum_{j=0}^{k} \frac{(-1)^{j}(n-j) !(-v) !}{j !(k-j) !(-v-j) ! 2^{k-j}}\right) z^{n-2 k}
\end{aligned}
$$

The inner sum in this expression can be expressed in two different ways (due to transformations relations) by the Jacobi polynomials $\mathrm{P}_{k}^{(\alpha, \beta)}(u)$ [3] [5] taken for argument $u=0$ or $u=3$ as follows 


$$
\begin{aligned}
\mathrm{He}_{n}^{v}(z) & =\sum_{k=0}^{\left[\frac{n}{2}\right]} \frac{(n-k) !}{(n-2 k) !} \mathrm{P}_{k}^{(-v-k, n+v-k)}(0) z^{n-2 k} \\
& =\sum_{k=0}^{\left[\frac{n}{2}\right]} \frac{(n-k) !}{(n-2 k) !} \frac{1}{2^{k}} \mathrm{P}_{k}^{(-v-k,-n-1)}(3) z^{n-2 k} .
\end{aligned}
$$

For the initial members $\operatorname{He}_{0}^{v}(z)$ of the sequences of polynomials (5.3) or (5.4) the sum consists only of the term to $k=0$ and due to $\mathrm{P}_{0}^{(\alpha, \beta)}(z)=1$ for arbitrary values of variable $z$ and parameters $(\alpha, \beta)$ we find

$$
\mathrm{He}_{0}^{v}(z)=1 \text {. }
$$

The given representations of $\mathrm{He}_{n}^{v}(z)$ can be proved, for example, by complete induction after derivation of the recurrence relations (6.4). It is remarkable that the upper index $v$ of the Associated Hermite polynomials denoting a parameter is only contained in the Jacobi polynomials in the representation (5.4). The Associated Hermite polynomials satisfy the following symmetry property

$$
\mathrm{He}_{n}^{v}(z)=(-\mathrm{i})^{n} \mathrm{He}_{n}^{-n-v}(\mathrm{i} z)=\mathrm{i}^{n} \mathrm{He}_{n}^{-n-v}(-\mathrm{i} z)=(-1)^{n} \mathrm{He}_{n}^{v}(-z) .
$$

Applying this identity and substituting $v \rightarrow v-1$ in (5.2) this formula can be written in the form

$$
\begin{aligned}
\mathrm{D}_{v-1-n}(z) & =\frac{(v-1-n) !}{(v-1) !}\left\{(-\mathrm{i})^{n} \mathrm{He}_{n}^{-v}(\mathrm{i} z) \mathrm{D}_{v-1}(z)-(-\mathrm{i})^{n-1} \mathrm{He}_{n-1}^{-v+1}(\mathrm{i} z) \mathrm{D}_{v}(z)\right\} \\
& =(-1)^{n} \frac{(-v) !}{(n-v) !}\left\{\operatorname{He}_{n}^{v-n}(z) \mathrm{D}_{v-1}(z)-\mathrm{He}_{n-1}^{v-n}(z) \mathrm{D}_{v}(z)\right\}, \quad(n \geq 0),
\end{aligned}
$$

which, in particular, is advantageous in the special cases of integer $v$, in particular, $v=0$ in which factors in front of the right-hand side of (5.2) become undetermined without limiting considerations or without using the Pochhammer symbol.

In the special case $v=0$ the involved Jacobi polynomials in (5.4) are evaluated to

$$
\mathrm{P}_{k}^{(-k, n-k)}(0)=\frac{1}{2^{k}} \mathrm{P}_{k}^{(-k,-n-1)}(3)=\frac{(-1)^{k} n !}{2^{k} k !(n-k) !},
$$

as one may see also from (5.3) and the sequence of polynomials $\mathrm{He}_{n}^{0}(z)$ possesses the form

$$
\mathrm{He}_{n}^{0}(z)=\sum_{k=0}^{\left[\frac{n}{2}\right]} \frac{(-1)^{k} n !}{k !(n-2 k) !} \frac{z^{n-2 k}}{2^{k}}=\frac{1}{(\sqrt{2})^{n}} \mathrm{H}_{n}\left(\frac{z}{\sqrt{2}}\right) \equiv \mathrm{He}_{n}(z) .
$$

This means that they possess a form of scaled Hermite polynomials $\mathrm{H}_{n}(z)$ which is usually denoted by $\mathrm{He}_{n}(z)$. In the Appendices $\mathrm{A}$ and $\mathrm{B}$ we give some initial members of the sequences $\mathrm{He}_{n}^{v}(z)$ for integer and for semi-integer $v$ in explicit form. In Appendix $\mathrm{C}$ we give explicit formulae for the Parabolic $\mathrm{Cy}$ linder functions $\mathrm{D}_{v}(z)$ with integer $v= \pm n$ related to the basic functions 
$\mathrm{D}_{0}(z)$ and $\mathrm{D}_{-1}(z)$. We mention that by means of the polynomials $\operatorname{He}_{n}(z)$ the operator disentanglement formula (4.8) can be represented in the form $(\alpha \rightarrow 2 \gamma)$

$$
\left(\gamma z-\beta \frac{\partial}{\partial z}\right)^{n}=\sum_{k=0}^{n} \frac{(-1)^{k} n !}{k !(n-k) !}(\sqrt{\gamma \beta})^{n-k} \mathrm{He}_{n-k}\left(\frac{\gamma z}{\sqrt{\gamma \beta}}\right)\left(\beta \frac{\partial}{\partial z}\right)^{k} .
$$

The right-hand side may be considered as disentanglement in normal ordering of the operators on the left-hand side. Other forms of operator disentanglement can be found in [14].

In general, the Parabolic Cylinder function $\mathrm{D}_{v}(z)$ can be composed according to (5.1) and (5.2) only from two basic Parabolic Cylinder functions where it is also possible to develop formulae with steps of $v$ greater than 1 . In special cases of zeros of one of the functions $\mathrm{D}_{v}(z)$ or $\mathrm{D}_{v-1}(z)$ in (5.1) and analogously in (5.2) the 3-term relations reduce to 2-term relations, for example, in case of a zero of $\mathrm{D}_{v-1}(z)$ for a certain $z=z_{0}$ according to

$$
\mathrm{D}_{v-1}\left(z_{0}\right)=0: \Rightarrow \mathrm{D}_{v+n}\left(z_{0}\right)=\mathrm{He}_{n}^{v}\left(z_{0}\right) \mathrm{D}_{v}\left(z_{0}\right) .
$$

Optimization problems for relative extrema in some problems may lead to the search for zeros of functions in series of the form $\sum_{n=0}^{\infty} c_{n} \mathrm{D}_{v+n}(z)$ with coefficients $c_{n}$ to determine and for such and also other cases it would be useful to know also generating functions for the Associated Hermite polynomials and in case of Bessel functions for the Lommel polynomials.

The use of two neighbored basic Parabolic Cylinder function as basis functions is particularly interesting for the irreducible representation of $\mathrm{D}_{v}(z)$ with integer indices $v= \pm n$ using in this case $\mathrm{D}_{0}(z)$ and $\mathrm{D}_{-1}(z)$ according to $(2.10)$ as such. This case concerns also the most important applications in quantum mechanics. In case of the irreducible representation of the Parabolic Cylinder functions with semi-integer indices one cannot find according to (2.9) two equally well appropriate neighbored basic functions but it is well possible that by choosing $\mathrm{D}_{1}(z)$ and $\mathrm{D}_{3}(z)$ as basis functions one may obtain more symmetric representations that we $\mathrm{e}^{-\overline{2}}$ do not try to do here.

\section{Recurrence Relations for the Associated Hermite Polynomials}

Recurrence relations for the Associated Hermite polynomials $\mathrm{He}_{n}^{v}(z)$ were derived in [6]. For some completeness of the description of these polynomials we partially repeat this here with some modifications.

As preparation for the calculation of relations for the Associated Hermite polynomials and of their differentiation it is useful to know some algebraic relations for the Jacobi polynomials. First of all these are recurrence relations. Since beside the variable $u$ we have in the Jacobi polynomials $\mathrm{P}_{n}^{(\alpha, \beta)}(u)$ two kinds of indices, the lower index $n=0,1,2, \cdots$ of the degree of the polynomials and two upper indices which may take on arbitrary real (or even complex) numbers there exists a great variety of such relations. We collect some of the most basic ones in 


\section{Appendix D.}

The derivation of the recurrence relations for the Associated Hermite polynomials can be made using the recurrence relations for the Parabolic Cylinder functions (4.7) which by the substitution $v \rightarrow v+n$ we write for this purpose in the form

$$
\mathrm{D}_{v+n+1}(z)=z \mathrm{D}_{v+n}(z)-(n+v) \mathrm{D}_{v+n-1}(z),
$$

and use now the representation (5.1) leading to the following form of the left-hand side of (6.1)

$$
\mathrm{D}_{v+n+1}(z)=\mathrm{He}_{n+1}^{v}(z) \mathrm{D}_{v}(z)-v \mathrm{He}_{n}^{v+1}(z) \mathrm{D}_{v-1}(z) .
$$

If we apply for the Parabolic Cylinder functions on the right-hand side of (6.1) the same representation (5.1) we find

$$
\begin{aligned}
\mathrm{D}_{v+n+1}(z)= & \left(z \mathrm{He}_{n}^{v}(z)-(n+v) \mathrm{He}_{n-1}^{v}(z)\right) \mathrm{D}_{v}(z) \\
& -v\left(z \mathrm{He}_{n-1}^{v+1}(z)-(n+v) \mathrm{He}_{n-2}^{v+1}(z)\right) \mathrm{D}_{v-1}(z) .
\end{aligned}
$$

Since the representation of the Parabolic Cylinder functions by two neighbored such functions is unique one finds by comparison of (6.2) with (6.3) the recurrence relation (compare also Drake [8] (Equation (1.1))

$$
\mathrm{He}_{n+1}^{v}(z)=z \mathrm{He}_{n}^{v}(z)-(n+v) \mathrm{He}_{n-1}^{v}(z) .
$$

For $v=0$ it provides the recurrence relation for the polynomials $\mathrm{He}_{n}(z)$ closely related to that for the usual Hermite polynomials $\mathrm{H}_{n}(z)$.

We now calculate

$$
\begin{aligned}
& \mathrm{He}_{n+1}^{v-1}(z)-\mathrm{He}_{n+1}^{v}(z) \\
& =\sum_{l=0}^{\left[\frac{n+1}{2}\right.} \frac{(n+1-l) !}{(n+1-2 l) !}\left(\mathrm{P}_{l}^{(-v-l+1, n+v-l)}(0)-\mathrm{P}_{l}^{(-v-l, n+v-l+1)}(0)\right) z^{n+1-2 l} \\
& =\sum_{l=1}^{\left[\frac{n+1}{2}\right.} \frac{(n+1-l) !}{(n+1-2 l) !} \mathrm{P}_{l-1}^{(-v-l+1, n+v-l+1)}(0) z^{n+1-2 l} \\
& =\sum_{k=0}^{\left[\frac{n-1}{2}\right.} \frac{(n-k) !}{(n-1-2 k) !} \mathrm{P}_{k}^{(-v-k, n+v-k)}(0) z^{n-1-2 k},
\end{aligned}
$$

where we applied the identity (D.3). If we now apply the recurrence relation (D.2) for the Jacobi polynomials $\mathrm{P}_{n}^{(\alpha, \beta)}(u)$ with fixed lower indices $n$ but varying upper indices with the substitutions $n \rightarrow k, \alpha \rightarrow-v-k, \beta \rightarrow n+v-k$ then from this follows

$$
(n-k) \mathrm{P}_{k}^{(-v-k, n+v-k)}(u)=(n+v) \mathrm{P}_{k}^{(-v-k, n-1+v-k)}(u)-v \mathrm{P}_{k}^{(-v-1-k, n+v-k)}(u) .
$$

Inserting this with $u=0$ in (6.5) we find an identity of the Associated Hermite polynomials in the form

$$
\begin{aligned}
\mathrm{He}_{n+1}^{v-1}(z)-\mathrm{He}_{n+1}^{v}(z) & =(n+v) \mathrm{He}_{n-1}^{v}(z)-v \mathrm{He}_{n-1}^{v+1}(z) \\
& =z \mathrm{He}_{n}^{v}(z)-\mathrm{He}_{n+1}^{v}(z)-v \mathrm{He}_{n-1}^{v+1}(z) .
\end{aligned}
$$


from which using (6.4) follows

$$
\operatorname{He}_{n+1}^{v-1}(z)+v \mathrm{He}_{n-1}^{v+1}(z)=z \mathrm{He}_{n}^{v}(z) .
$$

In next Section we derive formulae for the differentiation of the Associated Hermite polynomials from which we finally develop the differential equation for these polynomials.

\section{Derivative of the Associated Hermite Polynomials and Differential Equations}

By differentiation of the Associated Hermite polynomials $\mathrm{He}_{n}^{v}(z)$ in the representation (5.4) we find

$$
\frac{\partial}{\partial z} \mathrm{He}_{n}^{v}(z)=\sum_{k=0}^{\left[\frac{n-1}{2}\right]} \frac{(n-k) !}{(n-1-2 k) !} \mathrm{P}_{k}^{(-v-k, n+v-k)}(0) z^{n-1-2 k} .
$$

The right-hand side is the same as the last line on the right-hand side of (6.5) and therefore we may write the result of differentiation in the forms

$$
\begin{aligned}
\frac{\partial}{\partial z} \mathrm{He}_{n}^{v}(z) & =\mathrm{He}_{n+1}^{v-1}(z)-\mathrm{He}_{n+1}^{v}(z) \\
& =(n+v) \mathrm{He}_{n-1}^{v}(z)-v \mathrm{He}_{n-1}^{v+1}(z) \\
& =z \mathrm{He}_{n}^{v}(z)-\mathrm{He}_{n+1}^{v}(z)-v \mathrm{He}_{n-1}^{v+1}(z) .
\end{aligned}
$$

From this follows by index substitutions

$$
\begin{gathered}
\frac{\partial}{\partial z} \operatorname{He}_{n+1}^{v}(z)=(n+1+v) \mathrm{He}_{n}^{v}(z)-v \mathrm{He}_{n}^{v+1}(z), \\
\frac{\partial}{\partial z} \mathrm{He}_{n-1}^{v+1}(z)=\mathrm{He}_{n}^{v}(z)-\mathrm{He}_{n}^{v+1}(z) .
\end{gathered}
$$

By repeated differentiation using the representation in last line of (7.2) we obtain

$$
\begin{aligned}
\frac{\partial^{2}}{\partial z^{2}} \mathrm{He}_{n}^{v}(z) & =z \frac{\partial}{\partial z} \mathrm{He}_{n}^{v}(z)+\mathrm{He}_{n}^{v}(z)-\frac{\partial}{\partial z} \mathrm{He}_{n+1}^{v}(z)-v \frac{\partial}{\partial z} \mathrm{He}_{n-1}^{v+1}(z) \\
& =\left(z \frac{\partial}{\partial z}-n\right) \operatorname{He}_{n}^{v}(z)-2 v \frac{\partial}{\partial z} \operatorname{He}_{n-1}^{v+1}(z),
\end{aligned}
$$

where we used (7.3) for the transformation of the result. This means that the Associated Hermite polynomials satisfy the equation

$$
\left\{\frac{\partial^{2}}{\partial z^{2}}-z \frac{\partial}{\partial z}+n\right\} \mathrm{H} e_{n}^{v}(z)=-2 v \frac{\partial}{\partial z} \mathrm{He}_{n-1}^{v+1}(z) .
$$

For $v=0$ we obtain the differential equation for modified Hermite polynomials $\mathrm{H} e_{n}^{0}(z) \equiv \mathrm{He}_{n}(z)$.

The full elimination of the derivative $\frac{\partial}{\partial z} \mathrm{He}_{n-1}^{v+1}(z)$ by means of the last of Equations (7.3) provides

$$
\left\{\frac{\partial^{2}}{\partial z^{2}}-z \frac{\partial}{\partial z}+n+2 v\right\} \mathrm{He}_{n}^{v}(z)=2 v \mathrm{He}_{n}^{v+1}(z) .
$$


On the other side one obtains

$$
\left\{\frac{\partial^{2}}{\partial z^{2}}+z \frac{\partial}{\partial z}+n+2 v\right\} \operatorname{He}_{n}^{v}(z)=2(n+v) \mathrm{He}_{n}^{v-1}(z) .
$$

Under the assumption that both Equations (7.6) and (7.7) are correct (for (7.6) it is proved) one finds from these equations by forming the sum

$$
\left\{\frac{\partial^{2}}{\partial z^{2}}+n+2 v\right\} \mathrm{He}_{n}^{v}(z)=(n+v) \mathrm{He}_{n}^{v-1}(z)+v \mathrm{He}_{n}^{v+1}(z),
$$

and for the difference

$$
z \frac{\partial}{\partial z} \mathrm{He}_{n}^{v}(z)=(n+v) \mathrm{He}_{n}^{v-1}(z)-v \mathrm{He}_{n}^{v+1}(z) .
$$

Calculating the derivative from (5.4) we find

$$
z \frac{\partial}{\partial z} \operatorname{He}_{n}^{v}(z)=\sum_{k=0}^{\left[\frac{n}{2}\right]} \frac{(n-k) !}{(n-2 k) !}(n-2 k) \mathrm{P}_{k}^{(-v-k, n+v-k)}(0) z^{n-2 k} .
$$

Using herein the identity (D.8) of Appendix D with the substitutions $n \rightarrow k, \alpha \rightarrow-v-k, \beta \rightarrow n+v-k$ one obtains from (7.9)

$$
\begin{aligned}
& z \frac{\partial}{\partial z} \operatorname{He}_{n}^{v}(z) \\
& =\sum_{k=0}^{\left[\frac{n}{2}\right]} \frac{(n-k) !}{(n-2 k) !}\left((n+v) \mathrm{P}_{k}^{(-v+1-k, n+v-1-k)}(0)-v \mathrm{P}_{k}^{(-v-1-k, n+v+1-k)}(0)\right) z^{n-2 k} \\
& =(n+v) \mathrm{He}_{n}^{v-1}(z)-v \mathrm{He}_{n}^{v+1}(z) .
\end{aligned}
$$

Thus relations (7.8) and (7.9) and the equivalent relations (7.6) and (7.7) are proved.

Now we are able to derive the differential equations for which the Associated Hermite polynomials are solutions. Using (7.6) and (7.7) we have two possibilities with respect to the order of application which, clearly, have to lead to the same result. The first is

$$
\begin{aligned}
0 & =\left(\frac{\partial^{2}}{\partial z^{2}}+z \frac{\partial}{\partial z}+n+2(v+1)\right)\left\{\left(\frac{\partial^{2}}{\partial z^{2}}-z \frac{\partial}{\partial z}+n+2 v\right) \operatorname{He}_{n}^{v}(z)-2 v \mathrm{He}_{n}^{v+1}(z)\right\} \\
& =\left\{\left(\frac{\partial^{2}}{\partial z^{2}}+z \frac{\partial}{\partial z}+n+2(v+1)\right)\left(\frac{\partial^{2}}{\partial z^{2}}-z \frac{\partial}{\partial z}+n+2 v\right)-4 v(n+v+1)\right\} \operatorname{He}_{n}^{v}(z),
\end{aligned}
$$

and the second

$$
\begin{aligned}
0 & =\left(\frac{\partial^{2}}{\partial z^{2}}-z \frac{\partial}{\partial z}+n+2(v-1)\right)\left\{\left(\frac{\partial^{2}}{\partial z^{2}}+z \frac{\partial}{\partial z}+n+2 v\right) \mathrm{He}_{n}^{v}(z)-2(n+v) \mathrm{He}_{n}^{v-1}(z)\right\} \\
& =\left\{\left(\frac{\partial^{2}}{\partial z^{2}}-z \frac{\partial}{\partial z}+n+2(v-1)\right)\left(\frac{\partial^{2}}{\partial z^{2}}+z \frac{\partial}{\partial z}+n+2 v\right)-4(v-1)(n+v)\right\} \mathrm{He}_{n}^{v}(z) .
\end{aligned}
$$

By transition to normal ordering (all powers of $z$ stand in front of all powers 
of the differential operator $\frac{\partial}{\partial z}$ ) we find the following differential equation of 4-th order for which $\operatorname{He}_{n}^{v}(z)$ are solutions

$$
\left\{\frac{\partial^{4}}{\partial z^{4}}+\left(2(n+2 v)-z^{2}\right) \frac{\partial^{2}}{\partial z^{2}}-3 z \frac{\partial}{\partial z}+n(n+2)\right\} \mathrm{He}_{n}^{v}(z)=0 .
$$

In the special case $v=0$ and thus $\operatorname{He}_{n}^{0}(z) \equiv \mathrm{He}_{n}(z)$ the operator of this differential equation factorizes as follows

$$
\left(\frac{\partial^{2}}{\partial z^{2}}+z \frac{\partial}{\partial z}+n+2\right) \underbrace{\left(\frac{\partial^{2}}{\partial z^{2}}-z \frac{\partial}{\partial z}+n\right) \mathrm{He}_{n}(z)}_{=0}=0,
$$

where $\mathrm{He}_{n}(z)$ according to (7.5) satisfies already the shown differential equation of 2-nd order.

\section{Analogies and Differences between the Lommel Polynomials for the Bessel Functions to the Associated Hermite Polynomials for the Parabolic Cylinder Functions}

In the Introduction we already mentioned shortly the analogies between the Lommel polynomials $\mathrm{R}_{n}^{v}(z)$ in relation to the Bessel functions to the Associated Hermite polynomials $\operatorname{He}_{n}^{v}(z)$ in relation to the Parabolic Cylinder functions. We now come back to these analogies with more details.

The Bessel functions $\mathrm{J}_{v \pm n}(z)$ can be generated from two arbitrary basic functions $\mathrm{J}_{v}(z)$ and $\mathrm{J}_{v \mp 1}(z)$ according to the relations ([2] [3]; see also footnote 1)

$$
\begin{gathered}
\mathrm{J}_{v+n}(z)=\mathrm{R}_{n}^{v}(z) \mathrm{J}_{v}(z)-\mathrm{R}_{n-1}^{v+1}(z) \mathrm{J}_{v-1}(z), \\
\mathrm{J}_{v-n}(z)=(-1)^{n}\left\{\mathrm{R}_{n}^{-v}(z) \mathrm{J}_{v}(z)+\mathrm{R}_{n-1}^{1-v}(z) \mathrm{J}_{v+1}(z)\right\},
\end{gathered}
$$

where the sequences of polynomials $\mathrm{R}_{n}^{v}$ of $n$-th degree in variable $z^{-1}$, called Lommel polynomials, are given by

$$
\begin{aligned}
\mathrm{R}_{n}^{v}(z) & \equiv \sum_{k=0}^{\left[\frac{n}{2}\right]} \frac{(-1)^{k}(n-k) !(n+v-k-1) !}{k !(n-2 k) !(v+k-1) !}\left(\frac{2}{z}\right)^{n-2 k}, \quad(n \geq 0), \\
& =\underbrace{(v)_{n}}_{=\frac{(v+v-1) !}{(v-1) !}}\left(\frac{2}{z}\right)^{n}{ }_{2} \mathrm{~F}_{3}\left(\frac{1-n}{2},-\frac{n}{2} ;-n, v, 1-n-v ;-z^{2}\right), \quad(n \geq 0),
\end{aligned}
$$

where ${ }_{2} \mathrm{~F}_{3}\left(a_{1}, a_{2} ; c_{1}, c_{2}, c_{3} ; u\right)$ is a Hypergeometric function in its standard notation and $(v)_{n}$ the Pochhammer symbol. The polynomials $\mathrm{R}_{n}^{v}(z)$ possess the symmetry property

$$
\mathrm{R}_{n}^{v}(z)=(-1)^{n} \mathrm{R}_{n}^{1-n-v}(z)=\mathrm{R}_{n}^{1-n-v}(-z)=(-1)^{n} \mathrm{R}_{n}^{v}(-z) .
$$

The Bessel functions $\mathrm{J}_{v}(z)$ satisfy the differential equation 


$$
\begin{aligned}
0 & =\left\{\left(z \frac{\partial}{\partial z}\right)^{2}+z^{2}-v^{2}\right\} \mathbf{J}_{v}(z) \\
& =\left(\sqrt{\left(z \frac{\partial}{\partial z}\right)^{2}+z^{2}}+v\right)\left(\sqrt{\left(z \frac{\partial}{\partial z}\right)^{2}+z^{2}}-v\right) \mathbf{J}_{v}(z),
\end{aligned}
$$

with two commuting operators as factors in front. In contrast to the differential equation for the Parabolic Cylinder function the parameter $v$ of the Bessel function is here involved quadratically that invites to the given factorization.

The Parabolic Cylinder functions $\mathrm{D}_{v}(z)$ can be generated from two arbitrary basic functions $\mathrm{D}_{v}(z)$ and $\mathrm{D}_{v \mp 1}(z)$ according to the relations

$$
\begin{aligned}
\mathrm{D}_{v+n}(z)= & \operatorname{He}_{n}^{v}(z) \mathrm{D}_{v}(z)-v \mathrm{He}_{n-1}^{v+1}(z) \mathrm{D}_{v-1}(z), \quad(n=0,1,2, \cdots ; v \in \mathbb{R}), \\
\mathrm{D}_{v-n}(z)= & \frac{(v-n) !}{v !}\left\{(-\mathrm{i})^{n} \mathrm{He}_{n}^{-v-1}(\mathrm{i} z) \mathrm{D}_{v}(z)-(-\mathrm{i})^{n-1} \mathrm{He}_{n-1}^{-v}(\mathrm{i} z) \mathrm{D}_{v+1}(z)\right\}, \\
& (n=0,1,2, \cdots ; v \in \mathbb{R}) .
\end{aligned}
$$

where the sequences of polynomials $\mathrm{He}_{n}^{v}(z)$ of $n$-th degree are given by

$$
\begin{aligned}
\mathrm{He}_{n}^{v}(z) & =\sum_{k=0}^{\left[\frac{n}{2}\right]} \frac{(-1)^{k}}{(n-2 k) !}\left(\sum_{j=0}^{k} \frac{(n-j) !(v-1+j) !}{j !(k-j) !(v-1) ! 2^{k-j}}\right) z^{n-2 k} \\
& =\sum_{k=0}^{\left[\frac{n}{2}\right]} \frac{(n-k) !}{(n-2 k) !} \mathrm{P}_{k}^{(-v-k, n+v-k)}(0) z^{n-2 k} .
\end{aligned}
$$

They possess the symmetry property

$$
\mathrm{He}_{n}^{v}(z)=(-\mathrm{i})^{n} \mathrm{He}_{n}^{-n-v}(\mathrm{i} z)=\mathrm{i}^{n} \operatorname{He}_{n}^{-n-v}(-\mathrm{i} z)=(-1)^{n} \operatorname{He}_{n}^{v}(-z) .
$$

The Parabolic Cylinder functions $\mathrm{D}_{v}(z)$ satisfy the differential equation

$$
0=\left\{\frac{\partial^{2}}{\partial z^{2}}-\frac{z^{2}}{4}+v-\frac{1}{2}\right\} \mathrm{D}_{v}(z) \text {. }
$$

For the Lommel polynomials and for the Associated Hermite polynomials we know the explicit formulae ((8.2) and (5.3)). Since the formula for the Lommel polynomials involves the Hypergeometric function ${ }_{2} \mathrm{~F}_{3}\left(a_{1}, a_{2} ; c_{1}, c_{2}, c_{3} ; u\right)$ which satisfies a differential equation of 4 -th order the same should be true for the Lommel polynomials. However, we did not find it in literature and did not try to calculate it up to now. The derivation of the differential equation for the Associated Hermite polynomials (7.14) takes on an essential part of present paper.

Beside the mentioned similarities in both systems there are also essential differences. The most striking one is that $\operatorname{He}_{n}^{v}(z)$ are polynomials of $n$-th degree in $z$ whereas $\mathrm{R}_{n}^{v}(z)$ are polynomials of $n$-th degree in $\frac{1}{z}$, explicitly given in $(8.2)^{5}$. A certain feeling why this is so one may attain if one compares ${ }^{5}$ Therefore, a notation $\overline{\mathrm{R}}_{n}^{v}(z) \equiv \mathrm{R}_{n}^{v}\left(\frac{1}{z}\right)$ or similar would be better instead of the notation $\mathrm{R}_{n}^{v}(z) \equiv \mathrm{R}_{n, v}(z)$ for the Lommel polynomials based on literature. 
the two most important relations for the two systems of functions $\mathrm{D}_{v}(z)$ and $\mathrm{J}_{v}(z)$ beside the already written down differential equations that are the differentiations and the recurrence relations

$$
\begin{aligned}
& \frac{\partial}{\partial z} \mathrm{D}_{v}(z)=\frac{1}{2}\left(v \mathrm{D}_{v-1}(z)-\mathrm{D}_{v+1}(z)\right), \quad \frac{\partial}{\partial z} \mathrm{~J}_{v}(z)=\frac{1}{2}\left(\mathrm{~J}_{v-1}(z)-\mathrm{J}_{v+1}(z)\right), \\
& \mathrm{D}_{v}(z)=\frac{1}{z}\left(v \mathrm{D}_{v-1}(z)+\mathrm{D}_{v+1}(z)\right), \quad \mathrm{J}_{v}(z)=\frac{z}{2 v}\left(\mathrm{~J}_{v-1}(z)+\mathrm{J}_{v+1}(z)\right) .
\end{aligned}
$$

As essential difference we see that on the right-hand sides of the recurrence relations the variable $z$ stands for the Parabolic Cylinder functions $\mathrm{D}_{v}(z)$ in the denominator and for the Bessel functions $\mathrm{J}_{v}(z)$ in the numerator that explains this.

Other essential differences of the two considered systems of functions are the underlying symmetry groups. Let us make a few remarks about this in the next Section.

\section{Group-Theoretical Background of the Bessel Functions}

Whereas the group-theoretical background of the Parabolic Cylinder functions as shown is the Heisenberg-Weyl group $W(2)$ (see (4.3) and (4.4)) the group-theoretical background of the Bessel functions is the group of motions $M(2)$ of the two-dimensional Euclidean plane (Vilenkin [15], chap. IV). The generators of this group consist of two commuting translations $T_{2}$ in two independent directions of the plane and the one-parameter group of rotations $S O(2)$ in the plane which last does not commute with the translations (e.g., [15] [16] and [17]). Since this background is not so very well known let us give the most important relations in connection to the Bessel functions which show this. If we denote the two commuting operators of the Lie algebra of translations by $\left(Z_{1}, Z_{2}\right)$ and the operator of the rotation by $Z_{3}$ then we have the commutation relations for $\left(X_{1}, X_{2}, X_{3}\right)^{6}$

$$
\left[Z_{1}, Z_{2}\right]=0, \quad\left[Z_{2}, Z_{3}\right]=Z_{1}, \quad\left[Z_{3}, Z_{1}\right]=Z_{2} \text {. }
$$

We now form the new Lie-group operators $\left(A_{+}, A_{-}, A_{3}\right)$

$$
\begin{aligned}
& A_{+}=Z_{1}+\mathrm{i} Z_{2}, \quad A_{-}=Z_{1}-\mathrm{i} Z_{2}, \quad A_{3}=\mathrm{i} Z_{3}, \\
& Z_{1}=\frac{A_{+}+A_{-}}{2}, \quad Z_{2}=-\mathrm{i} \frac{A_{+}-A_{-}}{2}, \quad Z_{3}=-\mathrm{i} A_{3},
\end{aligned}
$$

which satisfy the commutation relations

$$
\left[A_{+}, A_{-}\right]=0, \quad\left[A_{3}, A_{+}\right]=+A_{+}, \quad\left[A_{3}, A_{-}\right]=-A_{-} .
$$

With respect to the Bessel functions $\mathrm{J}_{v}(z)$ we consider the following realization of the operators $\left(A_{+}, A_{-}, A_{3}\right)$

$$
A_{+}=\left(\frac{1}{z} \sqrt{\left(z \frac{\partial}{\partial z}\right)^{2}+z^{2}}-\frac{\partial}{\partial z}\right),
$$

${ }^{6}$ We use the notations of Barut and Raçzka [16] (chap. 14, \$3). Vilenkin [15] uses $\left(a_{1}, a_{2}, a_{3}\right)$ and Miller [17] $\left(P_{1}, P_{2}, M\right)$. 


$$
\begin{gathered}
A_{-}=\left(\frac{1}{z} \sqrt{\left(z \frac{\partial}{\partial z}\right)^{2}+z^{2}}+\frac{\partial}{\partial z}\right), \\
A_{3}=\sqrt{\left(z \frac{\partial}{\partial z}\right)^{2}+z^{2}},
\end{gathered}
$$

or equivalently for $\left(Z_{1}, Z_{2}, Z_{3}\right)$

$$
Z_{1}=\frac{1}{z} \sqrt{\left(z \frac{\partial}{\partial z}\right)^{2}+z^{2}}, \quad Z_{2}=\mathrm{i} \frac{\partial}{\partial z}, \quad Z_{3}=-\mathrm{i} \sqrt{\left(z \frac{\partial}{\partial z}\right)^{2}+z^{2}} .
$$

According to (8.4) the Bessel functions $\mathrm{J}_{v}(z)$ are eigenfunctions of the operator $\sqrt{\left(z \frac{\partial}{\partial z}\right)^{2}+z^{2}}$ to the eigenvalue $\pm v$ and one verifies

$$
\begin{gathered}
A_{+} \mathrm{J}_{v}(z)=\left(\frac{v}{z}-\frac{\partial}{\partial z}\right) \mathrm{J}_{v}(z)=\mathrm{J}_{v+1}(z), \\
A_{-} \mathrm{J}_{v}(z)=\left(\frac{v}{z}+\frac{\partial}{\partial z}\right) \mathrm{J}_{v}(z)=\mathrm{J}_{v-1}(z), \\
A_{3} \mathrm{~J}_{v}(z)=v \mathrm{~J}_{v}(z) .
\end{gathered}
$$

If we change the sign of $v$ then the transformation $v \rightarrow-v$ leads to the transformations $A_{ \pm} \rightarrow-A_{+}, A_{3} \rightarrow-A_{3}$ and the role of $A_{+}$and $A_{-}$becomes exchanged (beside sign changes) that preserves the commutation relations (9.3). The identity operator $I$ in the realization $I=1$ can be expressed by the Lie-algebra operators according to [16]

$$
\begin{aligned}
I & =\left(Z_{1}\right)^{2}+\left(Z_{2}\right)^{2}=\frac{1}{2}\left(A_{+} A_{-}+A_{-} A_{+}\right)=A_{+} A_{-}=A_{-} A_{+} \\
& =\left(\frac{1}{z} \sqrt{\left(z \frac{\partial}{\partial z}\right)^{2}+z^{2}}\right)^{2}-\frac{\partial^{2}}{\partial z^{2}} \\
& =\frac{1}{z^{2}}\left(\left(z \frac{\partial}{\partial z}\right)^{2}+z^{2}\right)-\frac{1}{z} \frac{\partial}{\partial z}-\frac{\partial^{2}}{\partial z^{2}}=1 .
\end{aligned}
$$

Similar to the system of Parabolic Cylinder functions $\mathrm{J}_{v \pm n}(z)$ connected with the Heisenberg-Weyl group $W(2)$ each (complete in some sense) system of Bessel functions $\mathrm{J}_{v \pm n}(z)$ with $0 \leq v<1$ and $n=0,1,2, \cdots$ realizes a countably infinite irreducible representation of the (inhomogeneous) group of motions $M(2)$ of the real two-dimensional Euclidean plane which can be represented as the semi-direct product $M(2)=T(2) \rrbracket S O(2)$ of the two-dimensional (commutative) translation group $T(2)$ with the one-parameter rotation group $S O(2)$ in the plane.

In application to systems of modified Bessel functions $\mathrm{I}_{v}(z)$ one has slightly to change the realizations of the lowering and raising operators and of the eigenvalue operator $\left(A_{+}, A_{-}, A_{3}\right)$ in comparison to (9.4) that we do not write down. 


\section{Conclusions}

In analogy to the known (but not well-known) relation of Bessel functions to Lommel polynomials we developed a similar concept for the relation of Parabolic Cylinder functions in the form $\mathrm{D}_{v}(z)$ to polynomials which we call Associated Hermite polynomials and denote them by $\operatorname{He}_{n}^{v}(z)$. These polynomials obey a fourth-order differential equation and their explicit form can be concisely expressed using the Jacobi polynomials $\mathrm{P}_{k}^{(\alpha, \beta)}(u)$ taken for argument $u$ equal to zero. Recurrence relations for these polynomials are derived. For the proof of the differential equation we give in Appendix D contiguous relations in the most symmetrical form concerning the parameters $(\alpha, \beta)$ and derive from them an identity (D.8) for Jacobi polynomials which is only true for argument $u$ equal to zero but likely can be generalized to general argument $u$ with coefficients depending on $u$. In Appendices A-B we write down their explicit form for some initial members $n$ and for some integer and semi-integer parameter $v$. In Appendix $\mathrm{C}$ we represent the Parabolic Cylinder functions $\mathrm{D}_{v}(z)$ for (positive and negative) integers $v$ by means of two basic such functions $\mathrm{D}_{0}(z)$ and $\mathrm{D}_{-1}(z)$ and the introduced Associated Hermite polynomials.

Our motivation for the introduction of the Associated Hermite polynomials in 2000 was analogies to the Lommel polynomials in relation to the Bessel functions. The Parabolic Cylinder functions together with the Associated Hermite polynomials possess as group-theoretical background the Heisenberg-Weyl group $W(2)$. For the Bessel functions together with the Lommel polynomials the group-theoretical background is the group of motions $M(2)$ with the two (commuting) translations and the one-parameter group of rotations in the plane. We made a comparison of formulae of both groups in relation to the Bessel and to the Parabolic Cylinder functions and gave explicitly the basic operators of their Lie algebras (in particular, lowering and raising operators) in representation related to these functions. Besides great analogies between the kind of formulae for these two sets of functions there are also essential differences. One such difference is that the Lommel polynomials are polynomials of variable $\frac{1}{z}$ whereas the Associated Hermite polynomials are polynomials in $z$. This is discussed and presented by the corresponding formulae.

We mention that in recent time the Parabolic Cylinder function are often used in a form denoted by $\mathrm{U}(\mu, z)$ closely and equivalently related to the here used older form $\mathrm{D}_{v}(z)$ by relation (2.8). Though some symmetries of $\mathrm{U}(\mu, z)$ are related to the parameter value $\mu=0$ and of $\mathrm{D}_{v}(z)$ to the value $v=-\frac{1}{2}$ the most important applications of the Parabolic Cylinder function are connected with integer values of $v$ in case of $\mathrm{D}_{v}(z)$ in opposition to semi-integer values of $\mu$ in case of $\mathrm{U}(\mu, z)$. Finally, the normalizable Parabolic Cylinder functions are representable as superpositions of $\mathrm{D}_{n}(z),(n=0,1,2, \cdots)$ that is very important for a rational representation of many formulae in quantum mechanics and we prefer this. 


\section{Conflicts of Interest}

The author declares no conflicts of interest regarding the publication of this paper.

\section{References}

[1] Von Lommel, E.C.J. (1871) Zur Theorie der Bessel'schen Funktionen. Mathematische Annalen, IV, 103-116. https://doi.org/10.1007/BF01443302

[2] Watson, G.N. (1944) (A Treatise on the) Theory of Bessel Functions. 2nd Edition, Cambridge University Press, Cambridge.

[3] Erdélyi, A. (1974) Higher Transcendental Functions, Vol. 2. Nauka, Moskva.

[4] Hurwitz, A. (1889) Über die Nullstellen der Bessel'schen Funktionen. Mathematische Annalen, XXXIII, 246-266.

[5] Szegö, G. (1959) Orthogonal Polynomials. 2nd Edition, American Mathematical Society, New York.

[6] Wünsche, A. (2003) Generalized Hermite Polynomials Associated with Functions of Parabolic Cylinder. Applied Mathematics and Computation, 141, 197-213. https://doi.org/10.1016/S0096-3003(02)00333-8

[7] Dattoli, G., Chiccoli, C., Lorenzutta, S., Maino, G. and Torre, A. (1994) Theory of Generalized Hermite Polynomials. Computers and Mathematics with Applications, 28, 71-83. https://doi.org/10.1016/0898-1221(94)00128-6

[8] Drake, D. (2009) The Combinatorics of Associated Hermite Polynomials. European Journal of Combinatorics, 30, 1005-1021. https://doi.org/10.1016/j.ejc.2008.05.009

[9] Erdélyi, A. (1974) Higher Transcendental Functions, Vol. 1. Nauka, Moskva.

[10] Temme, N.M. (2010) Parabolic Cylinder Functions, Chapter. 12. In: NIST Handbook of Mathematical Functions, Cambridge University Press, Cambridge, New York, 303-319.

[11] Olver, F.W.J., Lozier, D.W., Boisvert, R.F. and Clark, Ch.W. (Eds.) (2010) NIST Handbook of Mathematical Functions. Cambridge University Press, Cambridge, New York.

[12] Miller, J.C.P. (1964) Parabolic Cylinder Functions, Chapter. 19. In: Handbook of Mathematical Functions, National Bureau of Standards, Washington, 495-531.

[13] Abramowitz, M. and Stegum, I.A. (Eds.) (1964) Handbook of Mathematical Functions. National Bureau of Standards, Washington.

[14] Wünsche, A. (1999) Ordered Operator Expansions and Reconstruction from Ordered Moments. Journal of Optics B: Quantum and Semiclassical Optics, 1, 264-288. https://doi.org/10.1088/1464-4266/1/2/010

[15] Vilenkin, N.Ya. (1965) Spetsialnyje funkzii i teorija predstavlyenija grupp. [Special Functions and the Theory of Representations of Groups.] Nauka, Moskva.

[16] Barut, A.O. and Raçzka, R. (1977) Theory of Group Representations and Applications. PWN-Polish Scientific Publishers, Warszawa.

[17] Miller Jr., W. (1977) Symmetry and Separation of Variables. Addison-Wesley, Reading (Mass.).

[18] Wünsche, A. (2015) Operator Methods and SU(1,1) Symmetry in the Theory of Jacobi and of Ultraspherical Polynomials. Applied Mathematics, 7, 213-261. 


\section{Appendix A. Sequences of Associated Hermite Polynomials}

\section{$\mathrm{He}_{n}^{v}(z)$ with Integer Parameter $v$}

For convenience we compile here some first members of the sequences of polynomials form $\mathrm{He}_{n}^{v}(z)$ in explicit form.

The first 12 initial members of the sequence of polynomials $\mathrm{He}_{n}^{(1)}(z)$ are

$$
\begin{aligned}
& \operatorname{He}_{0}^{1}(z)=1, \\
& \operatorname{He}_{1}^{1}(z)=z \\
& \mathrm{He}_{2}^{1}(z)=z^{2}-2, \\
& \operatorname{He}_{3}^{1}(z)=z^{3}-5 z \\
& \mathrm{He}_{4}^{1}(z)=z^{4}-9 z^{2}+8=(z-1)(z+1)\left(z^{2}-8\right), \\
& \operatorname{He}_{5}^{1}(z)=z^{5}-14 z^{3}+33 z=z\left(z^{2}-3\right)\left(z^{2}-11\right), \\
& \operatorname{He}_{6}^{1}(z)=z^{6}-20 z^{4}+87 z^{2}-48, \\
& \operatorname{He}_{7}^{1}(z)=z^{7}-27 z^{5}+185 z^{3}-279 z \\
& \operatorname{He}_{8}^{1}(z)=z^{8}-35 z^{6}+345 z^{4}-975 z^{2}+384 \\
& \operatorname{He}_{9}^{1}(z)=z^{9}-44 z^{7}+588 z^{5}-2640 z^{3}+2895 z, \\
& \operatorname{He}_{10}^{1}(z)=z^{10}-54 z^{8}+938 z^{6}-6090 z^{4}+12645 z^{2}-3840, \\
& \operatorname{He}_{11}^{1}(z)=z^{11}-65 z^{9}+1422 z^{7}-12558 z^{5}+41685 z^{3}-35685 z
\end{aligned}
$$

The sequence of polynomials

$$
\mathrm{He}_{n}^{-1-n}(z)=\mathrm{i}^{n} \mathrm{He}_{n}^{1}(-\mathrm{i} z)=(-\mathrm{i})^{n} \mathrm{He}_{n}^{1}(\mathrm{i} z),
$$

is obtained from the sequence of polynomials $\mathrm{He}_{n}^{1}(z)$ in (A.1) by substituting all minus signs on the right-hand side by plus signs.

The first 12 members of the sequence of polynomials $\mathrm{He}_{n}^{0}(z) \equiv \mathrm{He}_{n}(z)$ are

$$
\begin{aligned}
& \operatorname{He}_{0}^{0}(z)=1 \\
& \operatorname{He}_{1}^{0}(z)=z \\
& \operatorname{He}_{2}^{0}(z)=z^{2}-1 \\
& \operatorname{He}_{3}^{0}(z)=z^{3}-3 z \\
& \operatorname{He}_{4}^{0}(z)=z^{4}-6 z^{2}+3 \\
& \mathrm{He}_{5}^{0}(z)=z^{5}-10 z^{3}+15 z \\
& \mathrm{He}_{6}^{0}(z)=z^{6}-15 z^{4}+45 z^{2}-15 \\
& \mathrm{He}_{7}^{0}(z)=z^{7}-21 z^{5}+105 z^{3}-105 z \\
& \mathrm{He}_{8}^{0}(z)=z^{8}-28 z^{6}+210 z^{4}-420 z^{2}+105 \\
& \operatorname{He}_{9}^{0}(z)=z^{9}-36 z^{7}+378 z^{5}-1260 z^{3}+945 z \\
& \operatorname{He}_{10}^{0}(z)=z^{10}-45 z^{8}+630 z^{6}-3150 z^{4}+4725 z^{2}-945, \\
& \operatorname{He}_{11}^{0}(z)=z^{11}-55 z^{9}+990 z^{7}-6930 z^{5}+17325 z^{3}-10395 z
\end{aligned}
$$

The sequence of polynomials $\mathrm{He}_{n}^{-n}(z)$ related to these polynomials are separately written down in explicit form. 
The first 12 members of the sequence of polynomials $\mathrm{He}_{n}^{-1}(z)$ are

$$
\mathrm{He}_{0}^{-1}(z)=1 \text {, }
$$$$
\mathrm{He}_{1}^{-1}(z)=z \text {, }
$$

$\mathrm{He}_{2}^{-1}(z)=z^{2}$,

$\mathrm{He}_{3}^{-1}(z)=z^{3}-z$

$\mathrm{He}_{4}^{-1}(z)=z^{4}-3 z^{2}$,

$\mathrm{He}_{5}^{-1}(z)=z^{5}-6 z^{3}+3 z$,

$\mathrm{He}_{6}^{-1}(z)=z^{6}-10 z^{4}+15 z^{2}$,

$\mathrm{He}_{7}^{-1}(z)=z^{7}-15 z^{5}+45 z^{3}-15 z$,

$\mathrm{He}_{8}^{-1}(z)=z^{8}-21 z^{6}+105 z^{4}-105 z^{2}$,

$\mathrm{He}_{9}^{-1}(z)=z^{9}-28 z^{7}+210 z^{5}-420 z^{3}+105 z$,

$\mathrm{He}_{10}^{-1}(z)=z^{10}-36 z^{8}+378 z^{6}-1280 z^{4}-945 z^{2}$,

$\mathrm{He}_{11}^{-1}(z)=z^{11}-45 z^{9}+630 z^{7}-3150 z^{5}+4725 z^{3}-945 z$.

Generally $\mathrm{He}_{n}^{-1}(z)$ is related to $\mathrm{He}_{n}^{0}(z)$ by

$$
\mathrm{He}_{n}^{-1}(z)=z \mathrm{He}_{n-1}^{0}(z) \text {. }
$$

The sequence of polynomials

$$
\mathrm{He}_{n}^{1-n}(z)=\mathrm{i}^{n} \mathrm{He}_{n}^{-1}(-\mathrm{i} z)=(-\mathrm{i})^{n} \mathrm{He}_{n}^{-1}(\mathrm{i} z),
$$

is obtained from the sequence of polynomials $\mathrm{He}_{n}^{-1}(z)$ in (4) by substituting all minus signs on the right-hand side by plus signs.

The first 12 members of the series of polynomials

$$
\mathrm{He}_{n}^{-n}(z)=\mathrm{i}^{n} \mathrm{He}_{n}^{0}(-\mathrm{i} z)=(-\mathrm{i})^{n} \mathrm{He}_{n}^{0}(\mathrm{i} z),
$$

are explicitly

$$
\begin{aligned}
& \mathrm{He}_{0}^{0}(z)=1 \\
& \mathrm{He}_{1}^{-1}(z)=z \\
& \mathrm{He}_{2}^{-2}(z)=z^{2}+1 \\
& \mathrm{He}_{3}^{-3}(z)=z^{3}+3 z \\
& \mathrm{He}_{4}^{-4}(z)=z^{4}+6 z^{2}+3 \\
& \mathrm{He}_{5}^{-5}(z)=z^{5}+10 z^{3}+15 z \\
& \mathrm{He}_{6}^{-6}(z)=z^{6}+15 z^{4}+45 z^{2}+15 \\
& \mathrm{He}_{7}^{-7}(z)=z^{7}+21 z^{5}+105 z^{3}+105 z \\
& \mathrm{He}_{8}^{-8}(z)=z^{8}+28 z^{6}+210 z^{4}+420 z^{2}+105 \\
& \mathrm{He}_{9}^{-9}(z)=z^{9}+36 z^{7}+378 z^{5}+1260 z^{3}+945 z \\
& \mathrm{He}_{10}^{-10}(z)=z^{10}+45 z^{8}+630 z^{6}+3150 z^{4}+4725 z^{2}+945 \\
& \mathrm{He}_{11}^{-11}(z)=z^{11}+55 z^{9}+990 z^{7}+6930 z^{5}+17325 z^{3}+10395 z
\end{aligned}
$$

The polynomials $\mathrm{He}_{n}^{-m}(z)$ with non-positive upper integer index $-m=0,-1,-2, \cdots$ 
are factorable and satisfy the following relation

$$
\mathrm{He}_{n}^{-m}(z)=\mathrm{He}_{m}^{-m}(z) \mathrm{He}_{n-m}^{0}(z), \quad(n-m \geq 0),
$$

for example

$$
\begin{aligned}
& \mathrm{He}_{n}^{-1}(z)=z \mathrm{He}_{n-1}^{0}(z), \\
& \mathrm{He}_{n}^{-2}(z)=\left(z^{2}+1\right) \mathrm{He}_{n-2}^{0}(z), \\
& \mathrm{He}_{n}^{-3}(z)=\left(z^{3}+3 z\right) \mathrm{He}_{n-3}^{0}(z), \\
& \mathrm{He}_{n}^{-4}(z)=\left(z^{4}+6 z^{2}+3\right) \mathrm{He}_{n-4}^{0}(z)
\end{aligned}
$$

The scaled Hermite Hermite polynomials $\mathrm{He}_{n}^{0}(z) \equiv \mathrm{He}_{n}(z)$ are connected with the usual Hermite polynomials $\mathrm{H}_{n}(z)$ by

$$
\mathrm{He}_{n}(z) \equiv \frac{1}{(\sqrt{2})^{n}} \mathrm{H}_{n}\left(\frac{z}{\sqrt{2}}\right), \Leftrightarrow \quad \mathrm{H}_{n}(z)=(\sqrt{2})^{n} \mathrm{He}_{n}(\sqrt{2} z),
$$

and play often a role in applications.

\section{Appendix B. Sequences of Associated Hermite Polynomials} $\mathrm{He}_{n}^{v}(z)$ with Semi-Integer Parameter $v$

The first 12 initial members of the sequence of polynomials $\mathrm{He}_{n}^{\frac{1}{2}}(z)$ are

$$
\begin{aligned}
& \operatorname{He}_{0}^{\frac{1}{2}}(z)=1, \\
& \operatorname{He}_{1}^{\frac{1}{2}}(z)=z \\
& \operatorname{He}_{2}^{\frac{1}{2}}(z)=z^{2}-\frac{3}{2} \\
& \operatorname{He}_{3}^{\frac{1}{2}}(z)=z^{3}-4 z \\
& \operatorname{He}_{4}^{\frac{1}{2}}(z)=z^{4}-\frac{15}{2} z^{2}+\frac{21}{4}, \\
& \operatorname{He}_{5}^{\frac{1}{2}}(z)=z^{5}-12 z^{3}+\frac{93}{4} z, \\
& \operatorname{He}_{6}^{\frac{1}{2}}(z)=z^{6}-\frac{35}{2} z^{4}+\frac{129}{2} z^{2}-\frac{231}{8}, \\
& \operatorname{He}_{7}^{\frac{1}{2}}(z)=z^{7}-24 z^{5}+\frac{285}{2} z^{3}-180 z, \\
& \operatorname{He}_{8}^{\frac{1}{2}}(z)=z^{8}-\frac{63}{2} z^{6}+\frac{1095}{4} z^{4}-\frac{2655}{4} z^{2}+\frac{3465}{16}, \\
& \operatorname{He}_{9}^{\frac{1}{2}}(z)=z^{9}-40 z^{7}+\frac{1911}{4} z^{5}-1875 z^{3}+\frac{27945}{16} z, \\
& \operatorname{He}_{10}^{\frac{1}{2}}(z)=z^{10}-\frac{99}{2} z^{8}+777 z^{6}-\frac{35805}{8} z^{4}+\frac{128835}{16} z^{2}-\frac{65835}{32}, \\
& \operatorname{He}_{11}^{\frac{1}{2}}(z)=z^{11}-60 z^{9}+1197 z^{7}-9492 z^{5}+\frac{443835}{16} z^{2}-\frac{81585}{4} z .
\end{aligned}
$$


The first 12 initial members of the sequence of polynomials $\operatorname{He}_{n}^{-\frac{1}{2}}(z)$ are

$$
\operatorname{He}_{0}^{-\frac{1}{2}}(z)=1
$$$$
\mathrm{He}_{1}^{-\frac{1}{2}}(z)=z \text {, }
$$

$\mathrm{He}_{2}^{-\frac{1}{2}}(z)=z^{2}-\frac{1}{2}$

$\mathrm{He}_{3}^{-\frac{1}{2}}(z)=z^{3}-2 z$

$\mathrm{He}_{4}^{-\frac{1}{2}}(z)=z^{4}-\frac{9}{2} z^{2}+\frac{5}{4}$

$\mathrm{He}_{5}^{-\frac{1}{2}}(z)=z^{5}-8 z^{3}+\frac{33}{4} z$

$\mathrm{He}_{6}^{-\frac{1}{2}}(z)=z^{6}-\frac{25}{2} z^{4}+\frac{57}{2} z^{2}-\frac{45}{8}$,

$\mathrm{He}_{7}^{-\frac{1}{2}}(z)=z^{7}-18 z^{5}+\frac{145}{2} z^{3}-51 z$,

$\mathrm{He}_{8}^{-\frac{1}{2}}(z)=z^{8}-\frac{49}{2} z^{6}+\frac{615}{4} z^{4}-\frac{945}{4} z^{2}+\frac{585}{16}$,

$\mathrm{He}_{9}^{-\frac{1}{2}}(z)=z^{9}-32 z^{7}+\frac{1155}{4} z^{5}-780 z^{3}+\frac{6705}{16} z$

$\mathrm{He}_{10}^{-\frac{1}{2}}(z)=z^{10}-\frac{81}{2} z^{8}+497 z^{6}-\frac{16695}{8} z^{4}+\frac{38835}{16} z^{2}-\frac{9945}{32}$,

$\operatorname{He}_{11}^{-\frac{1}{2}}(z)=z^{11}-50 z^{9}+801 z^{7}-4830 z^{5}+\frac{157395}{16} z^{3}-\frac{34335}{8} z$.

The first 12 initial members of the sequence of polynomials $\operatorname{He}_{n}^{-\frac{3}{2}}(z)$ are

$$
\begin{aligned}
& \operatorname{He}_{0}^{-\frac{3}{2}}(z)=1, \\
& \operatorname{He}_{1}^{-\frac{3}{2}}(z)=z, \\
& \operatorname{He}_{2}^{-\frac{3}{2}}(z)=z^{2}+\frac{1}{2}, \\
& \operatorname{He}_{3}^{-\frac{3}{2}}(z)=z^{3}, \\
& \operatorname{He}_{4}^{-\frac{3}{2}}(z)=z^{4}-\frac{3}{2} z^{2}-\frac{3}{4}, \\
& \operatorname{He}_{5}^{-\frac{3}{2}}(z)=z^{5}-4 z^{3}-\frac{3}{4} z, \\
& \operatorname{He}_{6}^{-\frac{3}{2}}(z)=z^{6}-\frac{15}{2} z^{4}+\frac{9}{2} z^{2}+\frac{21}{8}, \\
& \operatorname{He}_{7}^{-\frac{3}{2}}(z)=z^{7}-12 z^{5}+\frac{45}{2} z^{3}+6 z, \\
& \operatorname{He}_{8}^{-\frac{3}{2}}(z)=z^{8}-\frac{35}{2} z^{6}+\frac{255}{4} z^{4}-\frac{75}{4} z^{2}-\frac{231}{16},
\end{aligned}
$$




$$
\begin{aligned}
& \mathrm{He}_{9}^{-\frac{3}{2}}(z)=z^{9}-24 z^{7}+\frac{567}{4} z^{5}-165 z^{3}-\frac{855}{16} z, \\
& \mathrm{He}_{10}^{-\frac{3}{2}}(z)=z^{10}-\frac{63}{2} z^{8}+273 z^{6}-\frac{5145}{8} z^{4}+\frac{1395}{16} z^{2}+\frac{3465}{32} \\
& \mathrm{He}_{11}^{-\frac{3}{2}}(z)=z^{11}-40 z^{9}+477 z^{7}-1848 z^{5}+\frac{23835}{16} z^{3}+\frac{1125}{2} z
\end{aligned}
$$

\section{Appendix C. Sequence of Parabolic Cylinder Functions with Integer Indices}

The first 12 members of the sequence of Parabolic Cylinder functions with non-negative integer indices are

$$
\begin{aligned}
& \mathrm{D}_{0}(z)=\exp \left(-\frac{z^{2}}{4}\right) \\
& \mathrm{D}_{1}(z)=\exp \left(-\frac{z^{2}}{4}\right) z \\
& \mathrm{D}_{2}(z)=\exp \left(-\frac{z^{2}}{4}\right)\left(z^{2}-1\right), \\
& \mathrm{D}_{3}(z)=\exp \left(-\frac{z^{2}}{4}\right)\left(z^{3}-3 z\right), \\
& \mathrm{D}_{4}(z)=\exp \left(-\frac{z^{2}}{4}\right)\left(z^{4}-6 z^{2}+3\right), \\
& \mathrm{D}_{5}(z)=\exp \left(-\frac{z^{2}}{4}\right)\left(z^{5}-10 z^{3}+15 z\right), \\
& \mathrm{D}_{6}(z)=\exp \left(-\frac{z^{2}}{4}\right)\left(z^{6}-15 z^{4}+45 z^{2}-15\right), \\
& \mathrm{D}_{7}(z)=\exp \left(-\frac{z^{2}}{4}\right)\left(z^{7}-21 z^{5}+105 z^{3}-105 z\right), \\
& \mathrm{D}_{8}(z)=\exp \left(-\frac{z^{2}}{4}\right)\left(z^{8}-28 z^{6}+210 z^{4}-420 z^{2}+105\right), \\
& \mathrm{D}_{9}(z)=\exp \left(-\frac{z^{2}}{4}\right)\left(z^{9}-36 z^{7}+378 z^{5}-1260 z^{3}+945 z\right), \\
& \mathrm{D}_{10}(z)=\exp \left(-\frac{z^{2}}{4}\right)\left(z^{10}-45 z^{8}+630 z^{6}-3150 z^{4}+4725 z^{2}-945\right),\left(z^{11}-55 z^{9}+990 z^{7}-6930 z^{5}+17325 z^{3}-10395 z\right), \\
& 4
\end{aligned}
$$

The first 12 members of the sequence of Parabolic Cylinder functions with negative integer indices represented in the form (5.2) or better (5.7) with $v=0$ and using (2.10) are (definition of Complementary Error function $\operatorname{Erfc}(u) \equiv 1-\operatorname{Erf}(u))$ 


$$
\begin{aligned}
& \mathrm{D}_{-1}(z)=\frac{1}{0 !} \exp \left(\frac{z^{2}}{4}\right) \sqrt{\frac{\pi}{2}} \operatorname{Erfc}\left(\frac{z}{\sqrt{2}}\right), \\
& \mathrm{D}_{-2}(z)=\frac{1}{1 !}\left\{-\exp \left(\frac{z^{2}}{4}\right) \sqrt{\frac{\pi}{2}} \operatorname{Erfc}\left(\frac{z}{\sqrt{2}}\right) z+\exp \left(-\frac{z^{2}}{4}\right)\right\} \text {, } \\
& \mathrm{D}_{-3}(z)=\frac{1}{2 !}\left\{\exp \left(\frac{z^{2}}{4}\right) \sqrt{\frac{\pi}{2}} \operatorname{Erfc}\left(\frac{z}{\sqrt{2}}\right)\left(z^{2}+1\right)-\exp \left(-\frac{z^{2}}{4}\right) z\right\} \text {, } \\
& \mathrm{D}_{-4}(z)=\frac{1}{3 !}\left\{-\exp \left(\frac{z^{2}}{4}\right) \sqrt{\frac{\pi}{2}} \operatorname{Erfc}\left(\frac{z}{\sqrt{2}}\right)\left(z^{3}+3 z\right)+\exp \left(-\frac{z^{2}}{4}\right)\left(z^{2}+2\right)\right\} \text {, } \\
& \mathrm{D}_{-5}(z)=\frac{1}{4 !}\left\{\exp \left(\frac{z^{2}}{4}\right) \sqrt{\frac{\pi}{2}} \operatorname{Erfc}\left(\frac{z}{\sqrt{2}}\right)\left(z^{4}+6 z^{2}+3\right)-\exp \left(-\frac{z^{2}}{4}\right)\left(z^{3}+5 z\right)\right\} \\
& \mathrm{D}_{-6}(z)=\frac{1}{5 !}\left\{-\exp \left(\frac{z^{2}}{4}\right) \sqrt{\frac{\pi}{2}} \operatorname{Erfc}\left(\frac{z}{\sqrt{2}}\right)\left(z^{5}+10 z^{3}+15 z\right)\right. \\
& \left.+\exp \left(-\frac{z^{2}}{4}\right)\left(z^{4}+9 z^{2}+8\right)\right\} \\
& \mathrm{D}_{-7}(z)=\frac{1}{6 !}\left\{\exp \left(\frac{z^{2}}{4}\right) \sqrt{\frac{\pi}{2}} \operatorname{Erfc}\left(\frac{z}{\sqrt{2}}\right)\left(z^{6}+15 z^{4}+45 z^{2}+15\right)\right. \\
& \left.-\exp \left(-\frac{z^{2}}{4}\right)\left(z^{5}+14 z^{3}+33 z\right)\right\} \text {, } \\
& \mathrm{D}_{-8}(z)=\frac{1}{7 !}\left\{-\exp \left(\frac{z^{2}}{4}\right) \sqrt{\frac{\pi}{2}} \operatorname{Erfc}\left(\frac{z}{\sqrt{2}}\right)\left(z^{7}+21 z^{5}+105 z^{3}+105 z\right)\right. \\
& \left.+\exp \left(-\frac{z^{2}}{4}\right)\left(z^{6}+20 z^{4}+87 z^{2}+48\right)\right\} \text {, } \\
& \mathrm{D}_{-9}(z)=\frac{1}{8 !}\left\{\exp \left(\frac{z^{2}}{4}\right) \sqrt{\frac{\pi}{2}} \operatorname{Erfc}\left(\frac{z}{\sqrt{2}}\right)\left(z^{8}+28 z^{6}+210 z^{4}+420 z^{2}+105\right)\right. \\
& \left.-\exp \left(-\frac{z^{2}}{4}\right)\left(z^{7}+27 z^{5}+185 z^{3}+279 z\right)\right\} \text {, } \\
& \mathrm{D}_{-10}(z)=\frac{1}{9 !}\left\{-\exp \left(\frac{z^{2}}{4}\right) \sqrt{\frac{\pi}{2}} \operatorname{Erfc}\left(\frac{z}{\sqrt{2}}\right)\left(z^{9}+36 z^{7}+378 z^{5}+1260 z^{3}+945 z\right)\right. \\
& \left.+\exp \left(-\frac{z^{2}}{4}\right)\left(z^{8}+35 z^{6}+345 z^{4}+975 z^{2}+384\right)\right\} \text {, } \\
& \mathrm{D}_{-11}(z)=\frac{1}{10 !}\left\{\operatorname { e x p } ( \frac { z ^ { 2 } } { 4 } ) \sqrt { \frac { \pi } { 2 } } \operatorname { E r f c } ( \frac { z } { \sqrt { 2 } } ) \left(z^{10}+45 z^{8}\right.\right. \\
& \left.+630 z^{6}+3150 z^{4}+4725 z^{2}+945\right) \\
& \left.-\exp \left(-\frac{z^{2}}{4}\right)\left(z^{9}+44 z^{7}+588 z^{5}+2640 z^{3}+2895 z\right)\right\} \text {, } \\
& \mathrm{D}_{-12}(z)=\frac{1}{11 !}\left\{-\exp \left(\frac{z^{2}}{4}\right) \sqrt{\frac{\pi}{2}} \operatorname{Erfc}\left(\frac{z}{\sqrt{2}}\right)\left(z^{11}+55 z^{9}\right.\right. \\
& \left.+990 z^{7}+6930 z^{5}+17325 z^{3}+10395 z\right) \\
& \left.+\exp \left(-\frac{z^{2}}{4}\right)\left(z^{10}+54 z^{8}+938 z^{6}+6090 z^{4}+12645 z^{2}+3840\right)\right\} \text {. }
\end{aligned}
$$


In applications (e.g., quantum mechanics) one has often to do with the scaled Parabolic Cylinder functions $\mathrm{D}_{n}^{\prime}(z) \equiv(\sqrt{2})^{n} \mathrm{D}_{n}(\sqrt{2} z)$ which we do not explicitly write down.

\section{Appendix D. Some Algebraic Relations for the Jacobi Polynomials}

We collect here a few algebraic relations for the Jacobi polynomials which are interesting for the derivation of relations between the Associated Hermite polynomials.

The only general basic recurrence relation for the Jacobi polynomials with stable upper indices $(\alpha, \beta)$ is the three-term relation

$$
\begin{aligned}
0= & 2(n+1)(n+\alpha+\beta+1)(2 n+\alpha+\beta) \mathrm{P}_{n+1}^{(\alpha, \beta)}(u) \\
& -(2 n+\alpha+\beta+1)\left((2 n+\alpha+\beta)(2 n+\alpha+\beta+2) u+\alpha^{2}-\beta^{2}\right) \mathrm{P}_{n}^{(\alpha, \beta)}(u) \\
& +2(n+\alpha)(n+\beta)(2 n+\alpha+\beta+2) \mathrm{P}_{n-1}^{(\alpha, \beta)}(u),
\end{aligned}
$$

connecting neighbored terms with lower indices $(n+1, n, n-1)$ is well known (e.g., [3], chap. 10.8. (11)). In addition there are possible a number of so-called contiguous relations where the upper indices $(\alpha, \beta)$ are involved with differences by integers. We give in the following a few basic ones of them.

There exists the following recurrence relations with only one lower index $n$ but with unstable upper indices $(\alpha, \beta)$ and constant coefficients (see also [3] (10.8) Equations (34)-(37))

$$
(n+\alpha+\beta) \mathrm{P}_{n}^{(\alpha, \beta)}(u)=(n+\beta) \mathrm{P}_{n}^{(\alpha, \beta-1)}(u)+(n+\alpha) \mathrm{P}_{n}^{(\alpha-1, \beta)}(u),
$$

and the following recurrence relation with two lower indices $n$ and $n-1$ and unstable upper indices

$$
\mathrm{P}_{n-1}^{(\alpha, \beta)}(u)=\mathrm{P}_{n}^{(\alpha, \beta-1)}(u)-\mathrm{P}_{n}^{(\alpha-1, \beta)}(u) .
$$

Both can be directly proved using basic definitions of the Jacobi polynomials or their explicit representations (e.g., [5] [18]). A recurrence relation with only one lower index but contiguous upper indices and with coefficients which depend on the variable $u$ is

$$
\mathrm{P}_{n}^{(\alpha-1, \beta-1)}(u)=\frac{1-u}{2} \mathrm{P}_{n}^{(\alpha, \beta-1)}(u)+\frac{1+u}{2} \mathrm{P}_{n}^{(\alpha-1, \beta)}(u) .
$$

It also can be proved from the definition or from the basic explicit representations of the Jacobi polynomials. By linear combination of the above three contiguous relations which are complete as the fundamental ones we may obtain some other but no more fundamental forms. All these identities are true for general argument $u$.

We now derive an identity which is only true for the special argument $u=0$ of the Jacobi polynomials. Setting $u=0$ in the identity (D.4) we find

$$
\mathrm{P}_{n}^{(\alpha-1, \beta-1)}(0)=\frac{1}{2}\left(\mathrm{P}_{n}^{(\alpha, \beta-1)}(0)+\mathrm{P}_{n}^{(\alpha-1, \beta)}(0)\right) .
$$


and if we substitute first the parameter $\alpha \rightarrow \alpha+1$ and second the parameter $\beta \rightarrow \beta+1$ we obtain from (5) the two identities

$$
\begin{aligned}
& 2 \mathrm{P}_{n}^{(\alpha, \beta-1)}(0)=\mathrm{P}_{n}^{(\alpha+1, \beta-1)}(0)+\mathrm{P}_{n}^{(\alpha, \beta)}(0), \\
& 2 \mathrm{P}_{n}^{(\alpha-1, \beta)}(0)=\mathrm{P}_{n}^{(\alpha, \beta)}(0)+\mathrm{P}_{n}^{(\alpha-1, \beta+1)}(0) .
\end{aligned}
$$

The contiguous relation (D.2) specialized for argument $u=0$ can be written

$$
2(n+\alpha+\beta) \mathrm{P}_{n}^{(\alpha, \beta)}(0)=(n+\beta) 2 \mathrm{P}_{n}^{(\alpha, \beta-1)}(0)+(n+\alpha) 2 \mathrm{P}_{n}^{(\alpha-1, \beta)}(0) .
$$

Substituting now the Jacobi polynomials on the right-hand side according to the identities (D.6) one obtains a relation which by some shortening of expressions on both sides can be represented in the form

$$
(\alpha+\beta) \mathrm{P}_{n}^{(\alpha, \beta)}(0)=(n+\beta) \mathrm{P}_{n}^{(\alpha+1, \beta-1)}(0)+(n+\alpha) \mathrm{P}_{n}^{(\alpha-1, \beta+1)}(0) .
$$

As already announced by the derivation this identity is specific for the argument $u=0$ of the Jacobi polynomials and cannot be generalized to arbitrary $u \neq 0$ by simply substituting only the argument zero of the Jacobi polynomials by an arbitrary one.

We mention in addition the following general relation between Jacobi polynomials with different arguments [5] (chap. IV, (4.22.1))

$$
\mathrm{P}_{n}^{(\alpha, \beta)}(u)=\left(\frac{1+u}{2}\right)^{n} \mathrm{P}_{n}^{(\alpha,-2 n-\alpha-\beta-1)}\left(\frac{3-u}{1+u}\right)=(-1)^{n} \mathrm{P}_{n}^{(\beta, \alpha)}(-u) .
$$

It is applied in (5.4) to get two different representations of the formula for $\mathrm{He}_{n}^{v}(z)$. 